\title{
Algebraic Signal Processing Theory: 1-D Space
}

\author{
Markus Püschel, Senior Member, IEEE, and José M. F. Moura, Fellow, IEEE
}

\begin{abstract}
In our paper titled "Algebraic Signal Processing Theory: Foundation and 1-D Time" appearing in this issue of the IEEe Transactions on Signal Processing, we presented the algebraic signal processing theory, an axiomatic and general framework for linear signal processing. The basic concept in this theory is the signal model defined as the triple $(\mathcal{A}, \mathcal{M}, \Phi)$, where $\mathcal{A}$ is a chosen algebra of filters, $\mathcal{M}$ an associated $\mathcal{A}$-module of signals, and $\Phi$ is a generalization of the $z$-transform. Each signal model has its own associated set of basic SP concepts, including filtering, spectrum, and Fourier transform. Examples include infinite and finite discrete time where these notions take their well-known forms. In this paper, we use the algebraic theory to develop infinite and finite space signal models. These models are based on a symmetric space shift operator, which is distinct from the standard time shift. We present the space signal processing concepts of filtering or convolution, " $z$-transform," spectrum, and Fourier transform. For finite length space signals, we obtain 16 variants of space models, which have the 16 discrete cosine and sine transforms (DCTs/DSTs) as Fourier transforms. Using this novel derivation, we provide missing signal processing concepts associated with the DCTs/DSTs, establish them as precise analogs to the DFT, get deep insight into their origin, and enable the easy derivation of many of their properties including their fast algorithms.
\end{abstract}

Index Terms-Algebra, boundary condition, Chebyshev polynomials, convolution, discrete cosine transform (DCT), discrete sine transform (DST), Fourier transform, module, representation theory, shift, signal extension, signal model.

\section{INTRODUCTION}

$\mathbf{S}$ TANDARD linear signal processing (SP) considers signals indexed by time (discrete or continuous) and time-invariant systems or filters. Associated with SP is the time shift operator, abstractly defined (in discrete form) as

$$
q \diamond t_{n}=t_{n+1}
$$

The formulas for linear convolution and the discrete-time Fourier transform for infinite-length signals or for circular convolution and the discrete Fourier transform (DFT) for finitelength signals can be derived from this definition of the shift.

In this paper we show that an alternative linear SP framework can be derived from a different definition of the shift operator. This shift operates undirected or symmetrically in contrast to the directed operation of the time shift in (1). For this reason, we call it the space shift; it is abstractly defined as

$$
q \diamond t_{n}=\frac{1}{2}\left(t_{n-1}+t_{n+1}\right) \text {. }
$$

Manuscript received December 3, 2005; revised April 8, 2008. The associate editor coordinating the review of this manuscript and approving it for publication was Dr. Andrew C. Singer. This work was supported by NSF through awards 9988296, 0310941, and 0634967.

The authors are with the Department of Electrical and Computer Engineering, Carnegie Mellon University, Pittsburgh, PA 15213-3890 USA (e-mail: pueschel@ece.cmu.edu; moura@ece.cmu.edu).

Digital Object Identifier 10.1109/TSP.2008.925259
Accordingly, we derive for infinite- and finite-length signals the appropriate space SP notions including filtering or convolution, “ $z$-transforms," spectrum, Fourier transforms, frequency response, and others. In the finite case, we explain the need for boundary conditions and identify 16 "natural" choices that have the 16 discrete cosine and sine transforms (DCTs/DSTs) as Fourier transforms. This establishes the DCTs/DSTs as exact analogs of the DFT, a satisfying alternative to the original derivation of the DCTs and DSTs as approximations to the Karhunen-Loève transform of a stationary process [2], [3]. The complete set of DCTs/DSTs was defined in [4] without derivation or motivation. In this paper, we jointly refer to the DCTs and DST as discrete trigonometric transforms (DTTs) even though this class is actually larger (e.g., it contains the real DFT and discrete Hartley transform).

We note that in other areas such as dynamic systems, it is common to consider different notions of shift [5].

We develop space SP as an instantiation of the algebraic signal processing theory (ASP), a general and axiomatic theory of (linear) SP presented in [1] and [6]. The central object in ASP is the signal model, defined as a triple $(\mathcal{A}, \mathcal{M}, \Phi)$, where $\mathcal{A}$ is the filter space (an algebra), $\mathcal{M}$ the signal space (an $\mathcal{A}$-module), and $\Phi$ generalizes the concept of $z$-transform. Many signal models are in principle possible, each with its own SP notions, including filtering, spectrum, or Fourier transform. ASP establishes that for finite signals and shift-invariant models, $\mathcal{A}$ and $\mathcal{M}$ are polynomial algebras $\mathbb{C}[x] / p(x)$, i.e., spaces of polynomials with multiplication modulo a fixed polynomial. For example, for the finite time model, which has the DFT as Fourier transform, both take the form $\mathcal{A}=\mathcal{M}=\mathbb{C}[x] /\left(x^{n}-1\right)$.

In [1], we explained how to derive signal models from a definition of the shift. Application to the time shift (1) yielded the well-known infinite and finite time signal models. In this paper, we derive signal models from the space shift (2). We identify and define the $C$-transform as the appropriate " $z$-transform" and, for finite space signals, show that the 16 DTTs are the appropriate space Fourier transforms. As expected, the finite space signals models underlying the DTTs are again built from polynomial algebras. One application of the ASP interpretation of the DTTs is the easy derivation of many of their properties and and their fast algorithms [7]-[9].

The DCT, type 3, was related to a polynomial algebra in [10]; all DTTs of types 1-4 were related to polynomial algebras in [11]; see also [12]. In all cases, no connection to signal processing was established.

Organization: We start with a brief overview of ASP in Section II. The focus will be on finite shift-invariant signal models that are built from polynomial algebras. In Sections III and IV, we derive the infinite and finite space models. The finite case is worked out in greater detail since it provides the underpinning of the frequently used DTTs including many of 
their properties. An important variant of the DTTs, and their underlying signal models, is derived in Section V. Finally, we offer conclusions in Section VII.

\section{Algebraic Signal Processing Theory}

We introduce the necessary background on the ASP and show infinite and finite time signal processing as examples. For a complete and detailed introduction we refer the reader to [1], [6]. For brevity we will denote linear signal processing by SP.

\section{A. Signal Model}

Algebra (Filter Space): An algebra $\mathcal{A}$ is a vector space that is also a ring, i.e., it permits multiplication of elements and the distributivity law holds. Examples include the sets $\mathbb{C}, \mathbb{R}$ of complex or real numbers and the set of polynomials with complex coefficients $\mathbb{C}[x]$. In SP, the set of filters is commonly assumed to be an algebra, with the multiplication being the concatenation of filters. We denote elements of algebras with $h$, the common symbol for filters in SP.

Module (Signal Space): Given an algebra $\mathcal{A}$, an $\mathcal{A}$-module $\mathcal{M}$ is a vector space that permits an operation "." of $\mathcal{A}$ on $\mathcal{M}$ :

$$
h \cdot s \in \mathcal{M}, \quad \text { for } h \in \mathcal{A}, \quad s \in \mathcal{M} .
$$

Further, several properties such as the distributivity law have to hold [13]. In SP, the signal space is commonly assumed to be an $\mathcal{A}$-module, where $\mathcal{A}$ is the associated space of filters. The operation $\cdot$ denotes filtering; (3) ensures that filtering a signal $s \in \mathcal{M}$ with a filter $h \in \mathcal{A}$ yields again a signal.

A special case of a module is given by $\mathcal{M}=\mathcal{A}$ (equality as sets, not as algebraic structures) with the operation in (3) being the ordinary multiplication in $\mathcal{A}$. This module is called the regular module.

Spectrum, Frequency Response, Fourier Transform: For every given $\mathcal{A}$ and $\mathcal{M}$, there is an associated notion of spectrum, frequency response, and Fourier transform (if they exist). See [1] for details.

Signal Model: In applications, signals do not arise as elements of modules, but, in the discrete case considered here, as infinite or finite sequences of numbers, e.g., $\mathbf{s}=\left(\ldots, s_{-1}, s_{0}, s_{1}, \ldots\right) \in \mathbb{C}^{\mathbb{Z}}$ or $\mathbf{s}=\left(s_{0}, \ldots, s_{n-1}\right) \in \mathbb{C}^{n}$. The purpose of the signal model, introduced next, is to assign a filter algebra $\mathcal{A}$ and an $\mathcal{A}$-module $\mathcal{M}$ to such sequences. This way, filtering is automatically defined (the operation of $\mathcal{A}$ on $\mathcal{M})$, and we get access to the associated notion of spectrum and Fourier transform. In the definition, we assume complex signals, but other base fields can be chosen.

Definition 1 (Signal Model): Let $V \subseteq \mathbb{C}^{I}, I \subset \mathbb{Z}$, be a vector space. A signal model for $V$ is a triple $(\mathcal{A}, \mathcal{M}, \Phi)$, where $\mathcal{A}$ is an algebra, $\mathcal{M}$ is an $\mathcal{A}$-module, and $\Phi$ is a bijective (one-to-one and onto) linear mapping

$$
\Phi: V \rightarrow \mathcal{M}, \quad \mathbf{s} \mapsto s \in \mathcal{M}
$$

Example: Discrete Infinite Time: The abstract definition of the signal model is best illustrated by an example. Namely, the

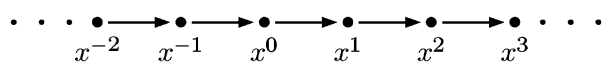

Fig. 1. Visualization of the infinite discrete time model (4) $\left(x=z^{-1}\right)$.

signal model commonly adopted for infinite length discrete time $\mathrm{SP}$ is given by (we set $x=z^{-1}$ )

$$
\begin{aligned}
\mathcal{A} & =\left\{\sum_{n \in \mathbb{Z}} h_{n} x^{n} \mid \mathbf{h}=\left(\ldots, h_{-1}, h_{0}, h_{1}, \ldots\right) \in \ell^{1}(\mathbb{Z})\right\} \\
\mathcal{M} & =\left\{\sum_{n \in \mathbb{Z}} s_{n} x^{n} \mid \mathbf{s}=\left(\ldots, s_{-1}, s_{0}, s_{1}, \ldots\right) \in \ell^{2}(\mathbb{Z})\right\} \\
\Phi & =\ell^{2}(\mathbb{Z}) \rightarrow \mathcal{M}, \quad \mathbf{s} \mapsto s=\sum_{n \in \mathbb{Z}} s_{n} x^{n} .
\end{aligned}
$$

The symbols $\ell^{1}(\mathbb{Z})$ and $\ell^{2}(\mathbb{Z})$ represent the set of infinite-length absolute summable and square summable (finite energy) sequences, respectively. As defined, $(\mathcal{A}, \mathcal{M}, \Phi)$ is a signal model for $V=\ell^{2}(\mathbb{Z})$ and $\Phi$ is just the ordinary $z$-transform. Note that in $\operatorname{ASP} \Phi(\mathbf{s})=s$ in (4) is primarily viewed as a formal series and not as a function. The idea is that $\Phi$ provides a basis for the coordinates $\mathbf{S}$ and gives convolution its desired form.

Shift and Shift-Invariance: In the algebraic theory, the shift (or shifts) is the chosen generator (or generators) of the filter algebra. This means that every filter can be expressed as a series or polynomial in the shift (or shifts). A signal model $(\mathcal{A}, \mathcal{M}, \Phi)$ has the shift-invariance property if and only if $\mathcal{A}$ is commutative. For example, the infinite discrete time model in (4) is shift-invariant, since the multiplication of Laurent series in $\mathcal{A}$ is commutative.

Visualization: Every (discrete) signal model implicitly fixes a basis of $\mathcal{M}$ via $\Phi$, such as $b=\left(\ldots, x^{-1}, x^{0}, x^{1}, \ldots\right)$ for the time model (4). The operation of the shift on this basis can be represented by a graph, which is called the visualization of the model (see [1] for a rigorous definition). The visualization of (4) is shown in Fig. 1. Intuitively, it is the structure imposed by the model on the signal values $\mathbf{s}$, which are associated with the nodes of the graph.

\section{B. Finite Shift-Invariant Signal Models}

We identify possible signal models for finite-length 1-D sequences $\mathbf{s}=\left(s_{0}, \ldots, s_{n-1}\right) \in V=\mathbb{C}^{n}$. In this case, $\operatorname{dim}(\mathcal{M})$, $\operatorname{dim}(\mathcal{A})<\infty$. If we require shift-invariance (i.e., $\mathcal{A}$ is commutative) and assume one shift, then $\mathcal{A}$ must be a polynomial algebra in one variable:

$$
\mathcal{A}=\mathbb{C}[x] / p(x)=\{q(x) \in \mathbb{C}[x] \mid \operatorname{deg}(q)<\operatorname{deg}(p)\} .
$$

Here, $p$ is an arbitrary but fixed polynomial, and addition and multiplication in $\mathcal{A}$ is defined modulo $p$. The shift in $\mathcal{A}$ is $x$.

In the following, we discuss signal models built from polynomial algebras and show the finite time model as an example. See [1] for more details. A good reference on polynomial algebras is [14].

Signal Model: We focus on a specific class of finite shiftinvariant 1-D signal models, namely, $\mathcal{A}$ chosen as in (5), $\mathcal{M}=$ $\mathcal{A}$ the regular $\mathcal{A}$-module, and we assume that $p$ is separable, i.e., 
has pairwise distinct zeros $\alpha=\left(\alpha_{0}, \ldots, \alpha_{n-1}\right)$. If we choose a basis $b=\left(p_{0}, \ldots, p_{n-1}\right)$ of polynomials in $\mathcal{M}$, then

$$
\begin{aligned}
& \mathcal{A}=\mathcal{M}=\mathbb{C}[x] / p(x), \\
& \Phi=\mathbb{C}^{n} \rightarrow \mathcal{M}, \quad \mathbf{s} \mapsto s=\sum_{0 \leq \ell<n} s_{\ell} p_{\ell}
\end{aligned}
$$

defines a signal model for $V=\mathbb{C}^{n}$. Filtering in this model is the multiplication $h(x) s(x) \bmod p(x)$ for $h \in \mathcal{A}, s \in \mathcal{M}$.

Signal Extension: Finite signals often arise because only a finite number of signal samples are available. How a finite signal continues beyond its domain is its signal extension.

Definition 2 (Signal Extension): Let $I \subset \mathbb{Z}$ and let $\mathbf{s}=$ $\left(s_{\ell}\right)_{\ell \in I} \in \mathbb{C}^{I}$. A (linear) signal extension of $\mathbf{s}$ is a series of linear combinations

$$
s_{k}=\sum_{\ell \in I} \beta_{k, \ell} s_{\ell}, \quad \text { for } k \notin I .
$$

If each summand contains at most one term, the signal extension is called monomial.

If we assume that the basis polynomials $p_{\ell}$ from $b$ in (6) are part of an infinite sequence $\left(p_{k}\right)_{k \in \mathbb{Z}}$, then (6) implicitly defines a signal extension for $\mathbf{s} \in \mathbb{C}^{n}$. It is given by reducing $p_{k}$ modulo $p$ and expressing the result in $b: p_{k} \equiv \sum_{0<\ell<n} \beta_{k, \ell} p_{\ell}$. Replacing $p$ by $s$ yields the signal extension in (7).

Spectrum, Fourier Transform, and Frequency Response: For the signal model (6), the spectral decomposition of $\mathcal{M}$, i.e., the Fourier transform, is given by the Chinese remainder theorem as

$$
\begin{aligned}
\Delta: \mathbb{C}[x] / p(x) & \rightarrow \mathbb{C}[x] /\left(x-\alpha_{0}\right) \oplus \ldots \oplus \mathbb{C}[x] /\left(x-\alpha_{n-1}\right) \\
s=s(x) & \mapsto\left(s\left(\alpha_{0}\right), \ldots, s\left(\alpha_{n-1}\right)\right) .
\end{aligned}
$$

$\Delta(s)$ is the spectrum of $s$. Further, $\Delta$ is linear ${ }^{1}$; hence, if we choose $b$ [which is fixed by $\Phi$ in (6)] as basis of $\mathcal{M}$ and $x^{0}=1$ as basis in each spectral component $\mathbb{C}[x] /\left(x-\alpha_{k}\right), \Delta$ is represented by the polynomial transform matrix

$$
\mathcal{P}_{b, \alpha}=\left[p_{\ell}\left(\alpha_{k}\right)\right]_{0 \leq k, \ell<n} .
$$

An arbitrary choice of bases $a_{k} x^{0}, a_{k} \in \mathbb{C}$, in the spectral components yields a scaled polynomial transform

$$
\operatorname{diag}\left(1 / a_{0}, \ldots, 1 / a_{n-1}\right) \cdot \mathcal{P}_{b, \alpha} .
$$

Any (scaled or not) polynomial transform is a Fourier transform for the signal model (6) and denoted with $\mathcal{F}$.

For a filter $h \in \mathcal{A},\left(h\left(\alpha_{0}\right), \ldots, h\left(\alpha_{n-1}\right)\right)$ is the frequency response of $h$. Filtering $h \cdot s(\bmod p)$ is equivalent to the pointwise multiplication $\left(h\left(\alpha_{0}\right) s\left(\alpha_{0}\right), \ldots, h\left(\alpha_{n-1}\right) s\left(\alpha_{n-1}\right)\right)$ in the spectral domain.

Filtering and Diagonalization Properties: For every filter $h \in \mathcal{A}$, filtering is a linear mapping on $\mathcal{M}$; thus, with respect to the basis $b=\left(p_{0}, \ldots, p_{n-1}\right)$ of $\mathcal{M}$ fixed by the model (6), $h$ is represented by an $n \times n$ matrix $M_{h}$. The mapping

$$
\phi: \mathcal{A} \rightarrow \mathbb{C}^{n \times n}, \quad h \mapsto \phi(h)=M_{h}
$$

is called the representation of $\mathcal{A}$ afforded by $\mathcal{M}$ with basis $b$. In particular, $\phi(x)$ is called the shift matrix. Filtering $h \cdot s$ becomes in coordinates the matrix-vector product $\phi(h) \mathbf{s}$.

\footnotetext{
${ }^{1}$ More precisely an $\mathcal{A}$-module homomorphism.
}

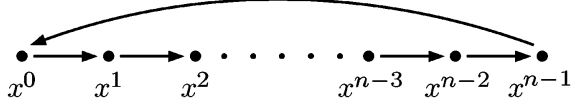

Fig. 2. Visualization of the finite discrete time model (12).

The matrices $\phi(h)$ are precisely those diagonalized by any Fourier transform $\mathcal{F}$ for the model. Specifically,

$$
\mathcal{F} \phi(h) \mathcal{F}^{-1}=\operatorname{diag}\left(h\left(\alpha_{0}\right), \ldots, h\left(\alpha_{n-1}\right)\right) .
$$

Visualization: The graph with adjacency matrix $\phi(x)$ (the shift matrix) is the visualization of the model (6).

Example: Discrete Finite Time: As an example we consider the commonly adopted signal model for discrete finite time, given by

$$
\begin{aligned}
& \mathcal{A}=\mathcal{M}=\mathbb{C}[x] /\left(x^{n}-1\right), \\
& \Phi=\mathbb{C}^{n} \rightarrow \mathcal{M}, \mathbf{s} \mapsto s=\sum_{0 \leq \ell<n} s_{\ell} x^{\ell} .
\end{aligned}
$$

We call $\Phi$ the finite $z$-transform. Note that the chosen basis (via $\Phi)$ is $b=\left(x^{0}, \ldots, x^{n-1}\right)$. Filtering in this model is polynomial multiplication $h(x) s(x)$ modulo $\left(x^{n}-1\right)$, which is equivalent to the circular convolution of $\mathbf{h}$ and $\mathbf{s}$. The signal extension is obtained by reducing $x^{k} \equiv x^{k \bmod n} \bmod \left(x^{n}-1\right)$ and is hence periodic and thus monomial.

The (polynomial) Fourier transform for the model (12) is readily computed via (9) as the DFT

$$
\mathcal{F}=\mathcal{P}_{b, \alpha}=\mathrm{DFT}_{n}=\left[\omega_{n}^{k \ell}\right]_{0 \leq k, \ell<n}, \quad \omega_{n}=e^{-2 \pi \sqrt{-1} / n} .
$$

For a filter $h \in \mathcal{A}$ the matrix $\phi(h)$ is a circulant matrix, which confirms the well-known property

$$
\operatorname{DFT}_{n} \phi(h) \operatorname{DFT}_{n}^{-1}=\operatorname{diag}\left(h\left(\omega_{n}^{0}\right), \ldots, h\left(\omega_{n}^{n-1}\right)\right) .
$$

The shift matrix $\phi(x)$ is the circular shift:

$$
\phi(x)=\left[\begin{array}{lll}
1 & & \\
& \ddots & \\
& & 1
\end{array}\right] .
$$

Thus, the visualization of the discrete finite time model is given by the directed circle in Fig. 2 that also captures the periodic signal extension. In words, applying a DFT to a signal $\mathbf{s} \in \mathbb{C}^{n}$ associates the values $s_{\ell}$ with the nodes of this graph, which is equivalent to imposing a periodic signal extension.

\section{Derivation of Signal Models}

In [1], we presented a procedure to derive infinite and finite signal models from an abstract definition of the shift operation. We used this procedure to derive the infinite and finite time models (4) and (12) from the standard time shift

$$
\text { time shift : } \quad q \diamond t_{n}=t_{n+1} \quad n \in \mathbb{Z} .
$$

displayed in Fig. 3 (top). Here the $t_{n}$ denote abstract time marks, $q$ is the shift operator, and $\diamond$ is the shift operation. 


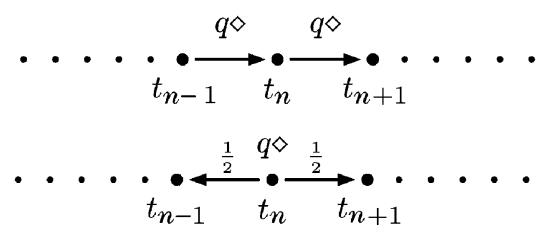

Fig. 3. Time shift (top) and space shift (bottom).

The procedure consists of three steps. First, the shift is defined in the abstract form shown in (13) and a $k$-fold shift $q_{k}$ is introduced through $q_{k} \diamond t_{n}=t_{n+k}$. This implies that $q_{k}=q^{k}$. Second, the shift operation is extended to linear combinations $\sum s_{n} t_{n}$ of the time marks and to linear combinations of $k$-fold shifts $q_{k}: \sum h_{k} q_{k}$. Third, the model is realized by setting $q=x$, replacing $\diamond$ with ordinary multiplication, and solving

$$
t_{n+1}=x \cdot t_{n}
$$

for $t_{n}$. Normalizing $t_{0}=1$ yields $t_{n}=x^{n}$ as unique solution. In the infinite case, convergence requirements lead to the model in (4). In the finite case, as was shown in [1], a boundary condition is needed to ensure that $\mathcal{M}$ becomes a module. This boundary condition determines the entire signal extension, and requiring a monomial signal extension (the simplest possible; see Definition 2) leads to $\mathcal{A}=\mathcal{M}=\mathbb{C}[x] /\left(x^{n}-a\right), a \in \mathbb{C}$. For $a=1$ this yields the finite time model (12).

In the following sections, we derive signal models for discrete infinite and finite space. These models are built using the same procedure but starting from a different definition of the shift.

\section{INFINITE 1-D SPACE MODELS}

Standard SP considers time-invariant systems, which implies the standard definition of the shift in (13). In this section and the next, we will use ASP to derive an SP framework for space SP as we refer to it. It is built from a different, symmetric definition of the shift. We have two motivations for this definition. The first is our goal to define the shift for signals for which there is no intrinsic sense of direction. These signals contrast with time signals, for which past, present, and future are inherent from the direction of time. The second reason is, as we will show, that our space shift definition leads to signal models that have the 16 DTTs as Fourier transforms. Thus, within ASP, time and space SP, the DFT and the DTTs become instantiations of one general framework. There will be many other benefits of this theoretical exercise as discussed later.

\section{A. Constructing the Signal Model} [1].

We follow the same steps as in the time model derivation in

Definition of the Shift: We consider discrete complex signals indexed by $\mathbb{Z}: s \in \mathbb{C}^{\mathbb{Z}}$; i.e., we consider the vector space $V=\mathbb{C}^{\mathbb{Z}}$. We define now space marks $t_{n}$ and an appropriate space shift operator $q$ and its operation $\diamond$ on the space marks. As mentioned above, $q$ should operate symmetrically. We adopt the definition

space shift : $\quad q \diamond t_{n}=\left(t_{n+1}+t_{n-1}\right) / 2, \quad n \in \mathbb{Z}$

visualized in Fig. 3 (bottom).
We proceed by extending the operator domain from $q$ to $k$-fold shift operators $q_{k}$. A natural definition of the $k$-fold space shift is

$$
q_{k} \diamond t_{n}=\left(t_{n+k}+t_{n-k}\right) / 2
$$

since $t_{n+k}$ and $t_{n-k}$ are those space marks at distance $k$ from $t_{k}$.

Here, we have the first interesting difference with respect to the time model derivation, since clearly $q_{k} \neq q^{k}$. Furthermore, (16) implies $q_{k}=q_{-k}$; hence, it is sufficient to consider only shift operators $q_{k}$ with $k \geq 0$. Thus, the natural representation of a filter will be $\sum_{k>0} h_{k} q_{k}$. The following lemma shows that the $q_{k}$ are given by the Chebyshev polynomials of the first kind $T_{k}$ in the variable $q$. The Chebyshev polynomials will play a central role in the definition of space models. For this reason, we provide the necessary background on four types of Chebyshev polynomials $T, U, V$, and $W$ in Appendix I, which we encourage the reader to briefly review at this point.

Lemma 3: The $k$-fold space shift operator is given by $q_{k}=$ $T_{k}(q)$.

Proof: Induction on $k$. By definition $q_{0}=1$, and $q_{1}=q=T_{1}(q)$. Also by definition, $q_{k+1} \diamond t_{n}=$ $\left(t_{n+k+1}+t_{n-k-1}\right) / 2=\left(t_{n+k+1}+t_{n+k-1}+t_{n-k+1}+\right.$ $\left.t_{n-k-1}\right) / 2-\left(t_{n+k-1}+t_{n-k+1}\right) / 2=2 q \diamond\left(t_{n+k}+t_{n-k}\right) / 2-$ $\left(t_{n+k-1}+t_{n-k+1}\right) / 2=\left(2 q q_{k}-q_{k-1}\right) \diamond t_{n}$, for $n \in \mathbb{Z}$. From the induction hypothesis, $q_{k}=T_{k}(q), q_{k-1}=T_{k-1}(q)$, and thus, using the recurrence of the Chebyshev polynomials [(43) in Appendix I], $q_{k+1}=T_{k+1}(q)$, as desired.

Linear Extension: To construct a linear signal model, we extend by linearity the operation of $q$ to the entire set $\mathcal{M}=\{s=$ $\left.\sum_{n \in \mathbb{Z}} s_{n} t_{n}\right\}$, namely as $\left.q \diamond s=\sum_{n \in \mathbb{Z}} s_{n}\left(q \diamond t_{n}\right)\right\}$, which can be evaluated. Similarly, we linearly extend the operator domain to $\mathcal{A}=\left\{h=\sum_{k \geq 0} h_{k} q_{k}\right\}=\left\{h=\sum_{k \geq 0} h_{k} T_{k}(q)\right\}$ using Lemma 3.

Realization: We determine a "realization" of the model introduced in the previous section. We set in (15) $q=x, \diamond=$., and determine polynomials $C_{n}$ that replace the space marks $t_{n}$ in (15), i.e., that satisfy

$$
x \cdot C_{n}=\left(C_{n+1}+C_{n-1}\right) / 2 .
$$

Since (17) is equivalent to (43) (in Appendix I), the solution is given by a sequence of Chebyshev polynomials.

We immediately notice differences with respect to the corresponding derivation in the time case. These differences are intrinsic to the space model.

- Equation (17) is a three-term recurrence for the space marks, whereas (14) is a two-term recurrence for the time marks.

- Only the $C_{n}, n \geq 0$, are linearly independent; the $C_{n}$, $n<0$, are polynomials in $x$ and can thus be expressed as linear combinations of $\left\{C_{n} \mid n \geq 0\right\}$. In other words, the realization of the space model introduces a starting point in space, given by $C_{0}=1$. Fixing $C_{1}$ determines the left boundary condition and the left signal extension.

- As a consequence, even after normalizing $C_{0}=1$, the sequence $C_{n}$ of Chebyshev polynomials is not uniquely determined. The degree of freedom is given by the choice of $C_{1}$ as a polynomial of degree 1 . 
TABLE I

REALIZATION OF THE ABStRact SpaCe Model

\begin{tabular}{lll}
\hline concept & abstract & realized \\
\hline shift operator & $q$ & $T_{1}(x)=x$ \\
shift operation & $\diamond$ & $\cdot$ \\
space mark & $t_{n}$ & $C_{n}$ \\
$k$-fold shift & $q_{k}=T_{k}(q)$ & $T_{k}(x)$ \\
space shift & $q \diamond t_{n}=\frac{1}{2}\left(t_{n+1}+t_{n-1}\right)$ & $x \cdot C_{n}=\frac{1}{2}\left(C_{n+1}+C_{n-1}\right)$ \\
signal & $\sum s_{n} t_{n}$ & $\sum s_{n} C_{n}(x)$ \\
filter & $\sum h_{k} T_{k}(q)$ & $\sum h_{k} T_{k}(x)$ \\
\hline
\end{tabular}

- Again, we note that in the time model, a $k$-fold shift operator is given by $x^{k}$ :

$$
x^{k} \cdot x^{n}=x^{n+k}
$$

in contrast to the space model, where, by Lemma 3, the $k$-fold shift operator is given by $T_{k}(x)$, independent of $C$ [see Lemma 14(iv) in Appendix I]:

$$
T_{k} \cdot C_{n}=\left(C_{n+k}+C_{n-k}\right) / 2 .
$$

As a result of this discussion, we obtain the spaces $\mathcal{A}=\{h=$ $\left.\sum_{k>0} h_{k} T_{k}\right\}$ and $\mathcal{M}=\left\{s=\sum_{n>0} s_{n} C_{n}\right\}$, i.e., the signal model that we obtain later will be only for right-sided sequences.

Table I shows the correspondence between abstract and realized concepts.

To ensure convergence, we would like to require as before $\mathbf{h} \in \ell^{1}(\mathbb{N})$ and $\mathbf{s} \in \ell^{2}(\mathbb{N})$. However, to prove convergence, we have first to choose proper boundary conditions, i.e., we have to choose the proper Chebyshev polynomials $C$. We analyze the boundary conditions in the next paragraph. This discussion has no counterpart in the derivation of the infinite time model in [1].

Left Boundary Condition and Left Signal Extension: The degree of freedom for choosing a Chebyshev sequence $C$, normalized by $C_{0}=1$, is given by the choice of $C_{1}$, or, equivalently, by the choice of $C_{-1}$, since the entire sequence is then obtained by applying the Chebyshev recursion (43) in both directions [see Lemma 14(i) in Appendix I]. Fixing either $C_{1}$ or $C_{-1}$ is equivalent to choosing a left boundary condition for the signal $\mathbf{s}=\left(s_{0}, s_{1}, \ldots\right)$. For example, setting $C_{1}=x$ implies $C_{-1}=x$, and thus $C_{-1}=C_{1}$, which imposes on the signal $\mathbf{s}$ the left boundary condition $s_{-1}=s_{1}$. Using Table VII, the corresponding sequence is $C=T$.

To determine the left boundary condition in the general case, we set $C_{0}=1$ and $C_{1}=a x+b, a \neq 0$ (to satisfy $\operatorname{deg}\left(C_{1}\right)=1$ ). Then, by applying (43) backwards, we get

$$
C_{-1}=2 x-(a x+b)=\frac{2-a}{a} C_{1}-\frac{2 b}{a} C_{0} .
$$

Since $C_{-1}$ is of degree at most 1 , every polynomial $C_{-n}, n>0$, obtained by the recursion (43), is of degree at most $n$, and thus a linear combination of the polynomials $C_{0}, \ldots, C_{n}$,

$$
C_{-n}=\sum_{0 \leq i \leq n} \beta_{i} \cdot C_{i} . \quad n>0
$$

This equation defines the left signal extension associated with the sequence $C$. On the other hand, by comparing the degrees of freedom, it is obvious that not every signal extension can be obtained by choosing a suitable boundary condition. Thus

$$
(C \Leftrightarrow \text { left boundary condition }) \Rightarrow \text { left signal extension. }
$$

For a generic left boundary condition, the left signal extension (20) has no simple structure; in particular, it is not monomial (see Definition 2). We determine now those left boundary conditions that yield a monomial left signal extension in (20). The answer is provided in the following lemma.

Lemma 4 (Monomial Left Signal Extension): Let $C=\left(C_{n} \mid n \in \mathbb{Z}\right)$ be a sequence of Chebyshev polynomials with $C_{0}=1$ and $\operatorname{deg}\left(C_{1}\right)=1$. Then the left signal extension associated with $C$ is monomial, i.e., every $C_{k}, k<0$, is a multiple of a $C_{n}, n \geq 0$, if and only if $C \in\{T, U, V, W\}$ (see Appendix I), i.e., $C_{-1} \in\left\{C_{1}, 0, C_{0},-C_{0}\right\}$, which implies the corresponding left boundary conditions $s_{-1} \in\left\{s_{1}, 0, s_{0},-s_{0}\right\}$.

Proof: If $C \in\{T, U, V, W\}$, then the assertion holds as shown in the "symmetry" column of Table VII. It remains to show the converse. We start with the generic left boundary condition in (19). Because the signal extension associated with $C$ is monomial, one of the two summands in (19) has to vanish.

Case 1: $C_{-1}$ is a multiple of $C_{0}$, i.e., constant. It follows $a=2, C_{1}=2 x+b, C_{-1}=-b, C_{-2}=-2 b x-1$. Now, either $C_{-2}$ is constant, i.e., $b=0$, which implies $C=U$, or $C_{-2}$ is a multiple of $C_{1}$, which implies $b= \pm 1$, or $C \in\{V, W\}$.

Case 2: $C_{-1}$ is a multiple of $C_{1}$. It follows $b=0, C_{1}=a x$, $C_{2}=2 a x^{2}-1, C_{-1}=(2-a) x, a \neq 2$, and $C_{-2}=2(2-$ a) $x^{2}-1$. Since $C_{-2}$ has to be a multiple of $C_{2}$, we get $a=1$ and thus $C=T$. This completes the proof.

The four boundary conditions in Lemma 4 are the discrete versions of the so-called Dirichlet boundary condition ("zero value") and von Neumann boundary condition ("zero slope") [15], [16]. In each case, the symmetry point is either a "whole" sample point, or a "half" sample point, i.e., is located between two sample points. In the literature, these four signal extensions are sometimes called: whole point symmetry (WS), whole point antisymmetry (WA), half point symmetry (HS), and half point antisymmetry (HA) [17].

For these four choices of boundary conditions, filtering, i.e., the multiplication $\sum_{n>0} h_{n} T_{n} \cdot \sum_{n>0} s_{n} C_{n}$ converges provided $\mathbf{h} \in \ell^{1}(\mathbb{N}), \mathbf{s} \in \ell^{2}(\mathbb{N})$ (see [6] for more details).

Resulting Infinite Space Models: We define four infinite space models $(\mathcal{A}, \mathcal{M}, \Phi)$ for $V=\ell^{2}(\mathbb{N})$. Namely, for $C \in\{T, U, V, W\}$

$$
\begin{gathered}
\mathcal{A}=\left\{h=\sum_{k \geq 0} h_{k} T_{k}(x) \mid \mathbf{h} \in \ell^{1}(\mathbb{N})\right\}, \\
\mathcal{M}=\left\{s=\sum_{n \geq 0} s_{n} C_{n}(x) \mid \mathbf{s} \in \ell^{2}(\mathbb{N})\right\}, \\
\Phi: \ell^{2}(\mathbb{N}) \rightarrow \mathcal{M}, \mathbf{s} \mapsto \sum_{n \geq 0} s_{n} C_{n}(x) .
\end{gathered}
$$

We call $\Phi$ the $C$-transform but will replace $C$ by either $T, U$, $V$, or $W$, when appropriate, and accordingly refer to the $T-, U$-, $V$-, or $W$-transform. 


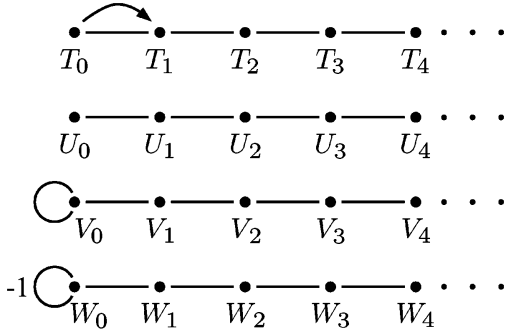

Fig. 4. Visualization of the four infinite space models for $C \in\{T, U, V, W\}$. The common edge scaling factor $1 / 2$ is omitted.

\section{B. Properties}

Each of these models has its associated notion of filtering, spectrum, frequency response, and Fourier transform as explained in [1]. We omit the details here since our focus are the finite space models that we will show to underly the DTTs.

The visualizations of the models are shown in Fig. 4 with a common scaling factors of $1 / 2$ omitted. The graphs are undirected, since they are space models. Namely, the space shift (Fig. 3 bottom) yields between each two space marks an edge in both directions. The behavior at the left edge is determined by the left boundary condition. Namely, $x C_{0}=\frac{1}{2}\left(C_{-1}+C_{1}\right)$ produces a directed edge to the (nonexistent) $C_{-1}$. In the first case, $C=T, T_{-1}=T_{1}$, and hence this edge is rerouted to $T_{1}$. In the second case, $C=U, U_{-1}=0$; hence, the edge vanishes.

\section{Finite 1-D Space Models ANd DTTS}

In this section, we derive finite versions of the space models in (21). As in the finite time model (12), these space models will have polynomial algebras as filter and signal spaces. This is not surprising as ASP explains that only those choices support shiftinvariance (Section II-A). We derive the finite space models in the same way as we derived the finite time model in [1], namely by requiring a monomial signal extension. However, in contrast with the time case, this signal extension will not be periodic but symmetric or antisymmetric with 16 choices. This is due to the different basis required after realizing the shift operation: $x^{\ell}$ supports the time shift, $C_{\ell}$ supports the space shift.

By applying the general theory from Section II-B, we will see that the Fourier transforms for the finite space models are precisely the 16 DTTs. There are various benefits to knowing these models. First, as application of the general theory in Section II-B, we obtain the appropriate notions of " $z$-transform," filtering or convolution, convolution theorems, spectrum and frequency response associated with the DTTs and can derive and explain many of their properties. Second, we establish that the DTTs are, in a rigorous sense, associated with the space shift, Fig. 3 (bottom), in the same way as the DFT is associated with the time shift. Third, knowing those signal models is the key to deriving and understanding the DTTs' fast algorithms [7], [9].

\section{A. Constructing the Signal Model}

Shift, Linear Extension, Realization: We consider a finite number of space marks $t_{0}, \ldots, t_{n-1}$ and adopt the space shift operator $q$ in Fig. 3 (bottom) and its realization by setting $q=x$, and hence $t_{k}=C_{k}$ (a generic sequence of Chebyshev polynomials), ${ }^{2}$ as derived in Section III-A. These definitions will need to be complemented by appropriate boundary conditions, as we discuss next.

Let $\mathbf{s}=\left(s_{0}, \ldots, s_{n-1}\right) \in \mathbb{C}^{n}$ be a finite sampled signal and $C$ a sequence of Chebyshev polynomials. A straightforward realization seems to lead to signals that are polynomials of the form $\sum_{0 \leq k<n} s_{k} C_{k}$. The set of these is the vector space $\mathbb{C}_{n}[x]$ of polynomials of degree less than $n$ (with basis polynomials $C_{k}$ ). However, this space is not closed under multiplication by the shift operator $x$, and thus it is not a module, which means filtering is not well-defined. In particular, the problem is that

$$
x \cdot C_{n-1}=\left(C_{n-2}+C_{n}\right) / 2 \notin \mathbb{C}_{n}[x]
$$

since $C_{n} \notin \mathbb{C}_{n}[x]$. Note that, in contrast to the time case [1], the left boundary does not impose any problems, since

$$
x \cdot C_{0}=\left(C_{-1}+C_{1}\right) / 2 \in \mathbb{C}_{n}[x] .
$$

Namely, the choice of $C$ already implies a left boundary condition via (19). So the remaining task is to determine the proper right boundary conditions.

Right Boundary Condition and Signal Extension: To solve the problem in (22), we introduce an equation

$$
C_{n}=r=\sum_{0 \leq k<n} \beta_{k} C_{k}, \quad \text { or } \quad C_{n}-r=0 .
$$

This imposes the same equation on the corresponding signal samples $s_{k}$ associated with $C_{k}$, namely

$$
s_{n}=\sum_{0 \leq k<n} \beta_{k} s_{k}
$$

which is the right boundary condition. As a consequence of (23), using the $k$-fold space shift operator $T_{k}$ (see Lemma 3 ), we get the series of equations for $k \geq 0$

$$
0=2 T_{k} \cdot 0 \equiv 2 T_{k}\left(C_{n}-r\right)=C_{n+k}+C_{n-k}-2 T_{k} r
$$

which determine the entire right signal extension. It is obtained by reducing $C_{n+k} \equiv 2 T_{k} r-C_{n-k}$ modulo $\left(C_{n}-r\right)$.

Algebraically, the right boundary condition replaces the vector space $\mathbb{C}_{n}[x]$ (with basis $b=\left(C_{0}, \ldots, C_{n-1}\right)$ ) by $\mathcal{M}=\mathbb{C}[x] /\left(C_{n}-r\right)$ (also with basis $\left.b\right)$, viewed as a regular module, i.e., the algebra is $\mathcal{A}=\mathcal{M}$. The natural basis in $\mathcal{A}$ is given by $\left(T_{0}, \ldots, T_{n-1}\right)$, regardless of the choice of $C$.

For a general choice of left boundary condition (given by the choice of $C$ ) and right boundary condition (given by the choice of $r$ ), the corresponding signal extension has a complicated structure. As before, we identify those boundary conditions that lead to a monomial signal extension. Lemma 4 gives already the proper left boundary conditions and shows that they are obtained by choosing $C \in\{T, U, V, W\}$. For the right boundary conditions, there are again four choices, which yields a total number of 16 possibilities-corresponding to the 16 types of DTTs as we will see below.

\footnotetext{
${ }^{2}$ We note that another realization is possible by setting $q$ not equal to $x$ However, the derived space models have two-dimensional spectral components, which is undesirable. See [6] for details.
} 
TABLE II

The 16 Polynomials $p$ Associated With the 16 Finite SPace Models. $C_{n}$ Has to BE REPLACED By $T_{n}, U_{n}, V_{n}, W_{n}$ TO OBtain Rows $1,2,3,4$, RESPECTIVELY

\begin{tabular}{lllll}
\hline$C_{n}$ & $C_{n}-C_{n-2}$ & $C_{n}$ & $C_{n}-C_{n-1}$ & $C_{n}+C_{n-1}$ \\
\hline$T_{n}$ & $2\left(x^{2}-1\right) U_{n-2}$ & $T_{n}$ & $(x-1) W_{n-1}$ & $(x+1) V_{n-1}$ \\
$U_{n}$ & $2 T_{n}$ & $U_{n}$ & $V_{n}$ & $W_{n}$ \\
$V_{n}$ & $2(x-1) W_{n-1}$ & $V_{n}$ & $2(x-1) U_{n-1}$ & $2 T_{n}$ \\
$W_{n}$ & $2(x+1) V_{n-1}$ & $W_{n}$ & $2 T_{n}$ & $2(x+1) U_{n-1}$ \\
\hline
\end{tabular}

Lemma 5 (Monomial Right Signal Extension): For a monomial left signal extension, let $C \in\{T, U, V, W\}$. The only four right boundary conditions that yield a monomial signal extension for $\mathcal{M}=\mathbb{C}[x] / p(x)$ are $C_{n}=C_{n-2}, C_{n}=0$, and $C_{n}=$ $\pm C_{n-1}$, which implies $p \in\left\{C_{n}-C_{n-2}, C_{n}, C_{n} \pm C_{n-1}\right\}$. These $16 p$ 's are shown in Table II.

Proof: Necessarily, the boundary condition has the form $C_{n}=a C_{k}, 0 \neq k<n$. By multiplying by $x$ on both sides, we obtain $C_{n+1}=a\left(C_{k+1}+C_{k-1}\right)-C_{n-1}$. We determine under which conditions the three summands on the right reduce to at most one summand.

Case 1: $k \neq n-1$ : Then either $a=0$, or $k=n-2$ and $a=1$.

Case 2: $k=n-1$ : Then $a C_{k+1}=a C_{n}=a^{2} C_{n-1}$ and thus $a= \pm 1$.

It remains to show that these four boundary conditions yield a monomial signal extension, which is done by induction. We omit the details.

The identities in Table II are obtained using Table VII in Appendix I and well-known trigonometric identities.

It is interesting to note that the right boundary conditions in Lemma 5 are the reflections of the left boundary conditions in Lemma 4.

Resulting Finite Space Models: We define 16 finite space models $(\mathcal{A}, \mathcal{M}, \Phi)$ for $V=\mathbb{C}^{n}$. Namely, for $C \in\{T, U, V, W\}$ and $p \in\left\{C_{n}-C_{n-2}, C_{n}, C_{n} \pm C_{n-1}\right\}$

$$
\begin{gathered}
\mathcal{A}=\mathbb{C}[x] / p(x)=\left\{h=\sum_{0 \leq k<n} h_{k} T_{k}(x) \mid h_{k} \in \mathbb{C}\right\} \\
\mathcal{M}=\mathbb{C}[x] / p(x)=\left\{s=\sum_{0 \leq k<n} s_{k} C_{k}(x) \mid s_{k} \in \mathbb{C}\right\} \\
\Phi: \mathbb{C}^{n} \rightarrow \mathcal{M}, \mathbf{s} \mapsto \sum_{0 \leq k<n} s_{k} C_{k}(x) .
\end{gathered}
$$

We call each $\Phi$ a finite $C$-transform, and replace $C$ with $T, U, V$, or $W$ if specified. Note that $\mathcal{A}=\mathcal{M}$ but the natural basis in $\mathcal{A}$ always consists of the $k$-fold space shifts $T_{k}$, independently of C.

Example: We choose the left boundary condition $s_{-1}=s_{0}$, i.e., $C_{-1}=C_{0}$, which is afforded by the base polynomials $C=V$. As right boundary condition, we choose $s_{n}=s_{n-1}$, i.e., $C_{n}=C_{n-1}$, which implies

$$
p=C_{n}-C_{n-1}=V_{n}-V_{n-1}=2(x-1) U_{n-1}
$$

TABLE III

EIGHT TYPES OF DCTS AND DSTS (UNSCALED) OF SIZE $n$. THE ENTRY AT ROW $k$ AND COLUMN $\ell$ IS GIVEN FOR $0 \leq k, \ell<n$

\begin{tabular}{cll}
\hline type & \multicolumn{1}{c}{ DCT } & \multicolumn{1}{c}{ DST } \\
\hline 1 & $\cos k \ell \frac{\pi}{n-1}$ & $\sin (k+1)(\ell+1) \frac{\pi}{n+1}$ \\
2 & $\cos k\left(\ell+\frac{1}{2}\right) \frac{\pi}{n}$ & $\sin (k+1)\left(\ell+\frac{1}{2}\right) \frac{\pi}{n}$ \\
3 & $\cos \left(k+\frac{1}{2}\right) \ell \frac{\pi}{n}$ & $\sin \left(k+\frac{1}{2}\right)(\ell+1) \frac{\pi}{n}$ \\
4 & $\cos \left(k+\frac{1}{2}\right)\left(\ell+\frac{1}{2}\right) \frac{\pi}{n}$ & $\sin \left(k+\frac{1}{2}\right)\left(\ell+\frac{1}{2}\right) \frac{\pi}{n}$ \\
5 & $\cos k \ell \frac{\pi}{n-\frac{1}{2}}$ & $\sin (k+1)(\ell+1) \frac{\pi}{n+\frac{1}{2}}$ \\
6 & $\cos k\left(\ell+\frac{1}{2}\right) \frac{\pi}{n-\frac{1}{2}}$ & $\sin (k+1)\left(\ell+\frac{1}{2}\right) \frac{\pi}{n+\frac{1}{2}}$ \\
7 & $\cos \left(k+\frac{1}{2}\right) \ell \frac{\pi}{n-\frac{1}{2}}$ & $\sin \left(k+\frac{1}{2}\right)(\ell+1) \frac{\pi}{n+\frac{1}{2}}$ \\
8 & $\cos \left(k+\frac{1}{2}\right)\left(\ell+\frac{1}{2}\right) \frac{\pi}{n+\frac{1}{2}}$ & $\sin \left(k+\frac{1}{2}\right)\left(\ell+\frac{1}{2}\right) \frac{\pi}{n-\frac{1}{2}}$ \\
\hline
\end{tabular}

using Table II. We obtain the associated signal model (the 2 in $p$ can be dropped)

$$
\begin{aligned}
& \mathcal{A}=\mathcal{M}=\mathbb{C}[x] /(x-1) U_{n-1}(x) \\
& \Phi: \mathrm{s} \mapsto s=\sum_{0 \leq \ell<n} s_{\ell} V_{\ell} .
\end{aligned}
$$

We will see later that the DCT, type 2, is a Fourier transform for this model.

Next, we apply the general theory from Section II-B to all 16 finite space models.

\section{B. Spectrum and Fourier Transform: DTTs}

We show that the 16 DTTs are Fourier transforms for the 16 finite space models (24). In doing so, we settle the question why there are 16 DTTs to begin with, as the original derivation of the full set of all 16 [4] does not provide an explanation.

The first and most important DTT is the DCT, type 2, introduced in [2] and used in the JPEG image compression standard. Table III gives the definitions of the nonorthogonal versions of the 16 DTTs. We note that the DTTs of type $1,4,5$, and 8 are symmetric, and that the DTTs of type 2 and 3, 6 and 7, respectively, are transposes of each other. We use Arabic instead of Roman numbers to denote the type following [16].

To compute the Fourier transform (8) of the finite space models (24) and its matrix form $\mathcal{F}$ in (9) or (10), we have to determine the zeros of the 16 polynomials in Table II, which can be done using Table VII in Appendix I. Instead of giving the details for all 16 cases, we consider the signal model (25) as a representative example and then state the result for all 16 DTTs. Note that the discussion is an application of the general theory in Section II-B.

Example: DCT, Type 2: The zeros of $(x-1) U_{n-1}(x)$ in (25) are given by $\alpha_{k}=\cos (k \pi / n), 0 \leq k<n$ (from Table VII in Appendix I). Hence, the Fourier transform for $\mathcal{M}$ is given by

$$
\begin{aligned}
\Delta: \mathbb{C}[x] /(x-1) U_{n-1}(x) & \rightarrow \bigoplus_{0 \leq k<n} \mathbb{C}[x] /\left(x-\alpha_{k}\right) \\
s=s(x) & \mapsto\left(s\left(\alpha_{0}\right), \ldots, s\left(\alpha_{n-1}\right)\right) .
\end{aligned}
$$

$\Delta(s)$ is the spectrum of the signal $s$ and $\left(h\left(\alpha_{0}\right), \ldots, h\left(\alpha_{n-1}\right)\right)$ is the frequency response of the filter $h \in \mathcal{A}$. 
TABLE IV

Overview of THE 16 DTTs AND THEIR Associated Signal Models. The LeFt Boundary Condition (Rows) Determines a SCALing Function $f(\cos \theta=x)$ AND the Basis $C \in\{T, U, V, W\}$ IN $\mathcal{M}=\mathbb{C}[x] / p(x)$ AND HenCE $\Phi$ The Right Boundary Condition (COlumns) Then Determines $p(x)$ (Given Below the DTT) AND Hence Also $\mathcal{A}=\mathcal{M}$

\begin{tabular}{lllllll}
\hline & $s_{n}-s_{n-2}$ & $s_{n}$ & $s_{n}-s_{n-1}$ & $s_{n}+s_{n-1}$ & $f$ & $C$ \\
\hline$s_{-1}=s_{1}$ & $\mathbf{D C T}-1$ & $\mathbf{D C T}-3$ & $\mathbf{D C T}-5$ & $\mathbf{D C T}-7$ & 1 & $T$ \\
& $2\left(x^{2}-1\right) U_{n-2}$ & $T_{n}$ & $(x-1) W_{n-1}$ & $(x+1) V_{n-1}$ & & \\
$s_{-1}=0$ & $\mathbf{D S T - 3}$ & $\mathbf{D S T - 1}$ & $\mathbf{D S T - 7}$ & $\mathbf{D S T - 5}$ & $\sin \theta$ & $U$ \\
& $2 T_{n}$ & $U_{n}$ & $V_{n}$ & $W_{n}$ & & \\
$s_{-1}=s_{0}$ & $\mathbf{D C T - 6}$ & $\mathbf{D C T - 8}$ & $\mathbf{D C T - 2}$ & $\mathbf{D C T - 4}$ & $\cos \frac{1}{2} \theta$ & $V$ \\
& $2(x-1) W_{n-1}$ & $V_{n}$ & $2(x-1) U_{n-1}$ & $2 T_{n}$ & & \\
$s_{-1}=-s_{0}$ & $\mathbf{D S T - 8}$ & $\mathbf{D S T - 6}$ & $\mathbf{D S T - 4}$ & $\mathbf{D S T - 2}$ & $\sin \frac{1}{2} \theta$ & $W$ \\
& $2(x+1) V_{n-1}$ & $W_{n}$ & $2 T_{n}$ & $2(x+1) U_{n-1}$ & & \\
\hline
\end{tabular}

In matrix form, the unique polynomial Fourier transform (9) for the signal model has entries

$$
V_{\ell}\left(\alpha_{k}\right)=\frac{1}{\cos k \pi /(2 n)} \cdot \cos k(\ell+1 / 2) \pi / n \text {. }
$$

We can scale these to cancel the denominator and get the matrix

$$
\begin{aligned}
\operatorname{diag}_{0 \leq k<n}(\cos k \pi /(2 n)) \cdot\left[V_{\ell}\left(\alpha_{k}\right)\right]_{0 \leq k, \ell<n} \\
=[\cos k(\ell+1 / 2) \pi / n]_{0 \leq k, \ell<n} \\
=\text { DCT- } 2_{n} .
\end{aligned}
$$

In words, the DCT- $2_{n}$ is a Fourier transform [namely a scaled polynomial transform (10)] for the signal model (25). The scaling diagonal in (27) shows the basis chosen on the right-hand side of (26), namely $1 /(\cos k \pi /(2 n))$ in the $k$ th spectral component $\mathbb{C}[x] /(x-\cos k \pi / n), 0 \leq k<n$.

All DTTs: Similar computations for all 16 cases establishes the 16 DTTs as Fourier transforms for the 16 finite $C$-transforms.

Theorem 6 (DTTs and Polynomial Algebras): The 16 DTTs are the Fourier transforms for the 16 finite space models (24). The correspondence is given in Table IV as follows. Let $(\mathcal{A}, \mathcal{M}, \Phi)$ be a finite space model with $\mathcal{M}=\mathbb{C}[x] / p$ with basis $b=\left(C_{0}, \ldots, C_{n-1}\right)$. The choice of $C$ (rows of Table IV) determines the left boundary condition and a scaling function $f$. The choice of right boundary condition (columns $2-5$ in Table IV) then determines the polynomial $p$, given at the intersection of row and column. The corresponding DTT is given above $p$. More specifically, assume $\alpha=\left(\alpha_{0}, \ldots, \alpha_{n-1}\right)$ are the zeros of $p$. All $\alpha_{k}$ have the form $\cos r_{k} \pi, r_{k} \in[0, \pi]$ (see Table VII in the Appendix I), and $\alpha$ is ordered by increasing $r_{k}$. Then

$$
\operatorname{DTT}_{n}=\operatorname{diag}_{0 \leq k<n}\left(f\left(\alpha_{k}\right)\right) \cdot \mathcal{P}_{b, \alpha}
$$

i.e., $\mathrm{DTT}_{n}$ is a scaled polynomial transform and thus a Fourier transform for the associated signal model (see Section II-B). Equation (28) implies that the chosen basis in the spectral component $\mathbb{C}[x] /\left(x-\alpha_{k}\right)$ is $1 / f\left(\alpha_{k}\right), 0 \leq k<n$.

The DCT, type 3 , was implicitly recognized as a polynomial transform in [10]. The DCTs and DSTs of types 1-4 where recognized as (scaled) polynomial transforms in [11]. In both cases no connection to signal processing was established. The original derivation of the DCT, type 2, in [2] mentions Chebyshev polynomials but does not make use of this fact nor connects to algebra.

Polynomial DTTs: Theorem 6 shows that each DTT is a Fourier transform for a finite space model but in general not the corresponding polynomial transform. Thus, we now associate to each DTT its polynomial transform $\mathcal{P}_{b, \alpha}$ obtained by omitting the scaling factors in (28).

Definition 7 (Polynomial DTTs): Let $\mathrm{DTT}_{n}$ be given. We call the unique polynomial transform $\mathcal{P}_{b, \alpha}$ associated with $\mathrm{DTT}_{n}$ by (28) the "polynomial DTT" and denote it with $\overline{\mathrm{DTT}}_{n}$. Thus, (28) can be rewritten as

$$
\mathrm{DTT}_{n}=\operatorname{diag}_{0 \leq k<n}\left(f\left(\alpha_{k}\right)\right) \cdot \overline{\mathrm{DTT}}_{n} .
$$

We have DTT $=\overline{\text { DTT }}$ if and only if DTT appears in the first row of Table IV, i.e., if DTT $\in\{$ DCT-1, DCT-3, DCT-5, DCT-7 $\}$.

The polynomial DTTs will play an important role in the derivation of fast DTT algorithms [7]. Also, in some cases the polynomial DTTs have a lower complexity than the actual DTT. This makes them a candidate for applications in which the DTT is followed by scaling (such as JPEG compression).

Remarks and Observations: For each DTT, we have three relevant versions. First, the polynomial version $\overline{\mathrm{DTT}}$, which is the unique polynomial transform for its associated signal model (see Definition 7 above). Second, the unscaled or natural version, which has pure cosines (or sines) as entries (see Table III). Third, the orthogonal version, which arises from the other two by suitable scaling of rows and columns, i.e., by slightly adjusting the signal model (explained below in Section IV-E).

The 16 DTTs can be divided into four groups of four each with respect to the polynomial $p$ in the associated module $\mathbb{C}[x] / p$ (see Table IV). For example, the " $T$-group" comprises all DTTs of types 3 and 4, which have the same module $\mathcal{M}=\mathbb{C}[x] / T_{n}$. The modules within the other groups differ slightly, e.g., in the $U$-group that comprises the DTTs on the main diagonal in Table IV. The difference between the DTTs within the same group is the choice of basis, which is one of $T, U, V, W$. As a consequence, these transforms can be converted into each other using a sparse base change (explained in Section IV-F). 


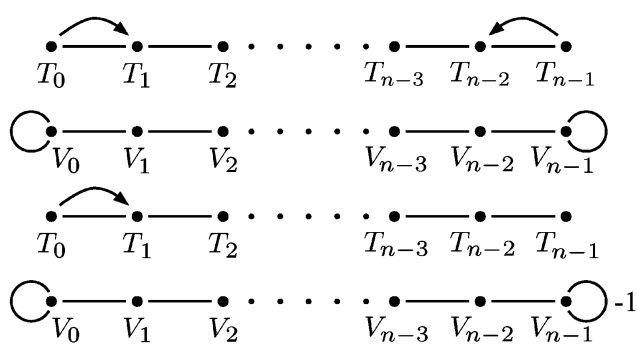

Fig. 5. Visualizations of the finite space models associated with the DCTs of type 1-4 (from top to bottom) and size $n$. A common edge scaling factor of $1 / 2$ has been omitted.

TABLE V

The VAlues $\beta_{1}, \beta_{2}, \beta_{3}, \beta_{4}$ FROM (30) FOR THe Four ResPeCtive Choices OF LEFT AND RIGHT BOUNDARY CONDITION

\begin{tabular}{|c|c|c|c|c|c|}
\hline left boundary condition & $\beta_{1}$ & $\beta_{2}$ & right boundary condition & $\beta_{3}$ & $\beta_{4}$ \\
\hline$s_{-1}=s_{1}$ & 0 & 2 & $s_{n}=s_{n-2}$ & 2 & 0 \\
\hline$s_{-1}=0$ & 0 & 1 & $s_{n}=0$ & 1 & 0 \\
\hline$s_{-1}=s_{0}$ & 1 & 1 & $s_{n}=s_{n-1}$ & 1 & 1 \\
\hline$s_{-1}=-s_{0}$ & -1 & 1 & $s_{n}=-s_{n-1}$ & 1 & -1 \\
\hline
\end{tabular}

\section{Visualization}

The right boundary conditions for the 16 finite space models (24) are precisely the mirrored left boundary conditions that occurred already in Fig. 4. This makes it easy to obtain the visualizations for (24). For example, Fig. 5 shows the cases associated with the DCTs of type $1-4$.

More formally, consider the model (25) as example. To obtain the visualization, we have to compute the shift matrix $\phi(x)$. From $x V_{0}=\frac{1}{2} V_{0}+\frac{1}{2} V_{1}, x V_{i}=\frac{1}{2} V_{i-1}+\frac{1}{2} V_{i+1}, 1 \leq i<n-1$, and $x V_{n-1}=\frac{1}{2} V_{n-2}+\frac{1}{2} V_{n} \equiv \frac{1}{2} V_{n-2}+\frac{1}{2} V_{n-1} \bmod \left(V_{n}-\right.$ $\left.V_{n-1}\right)$, it follows that

$$
\phi(x)=\frac{1}{2} \cdot\left[\begin{array}{lllll}
1 & 1 & & & \\
1 & 0 & \cdot & & \\
0 & 1 & \cdot & 1 & \\
& & \cdot & 0 & 1 \\
& & & 1 & 1
\end{array}\right] .
$$

This is precisely the adjacency matrix of the second graph in Fig. 5 associated with the DCT of type 2. In words, applying the DCT-2 to a signal s implicitly imposes the structure of this graph on that signal.

For an arbitrary finite space model (24), $\phi(x)$ takes the form

$$
\phi(x)=\frac{1}{2} \cdot\left[\begin{array}{ccccc}
\beta_{1} & 1 & & & \\
\beta_{2} & 0 & \cdot & & \\
0 & 1 & \cdot & 1 & \\
& & \cdot & 0 & \beta_{3} \\
& & & 1 & \beta_{4}
\end{array}\right]
$$

with the $\beta_{i}$ shown in Table V.

\section{Filtering and Diagonalization Properties}

Consider a finite space model (24) with $\mathcal{A}=\mathcal{M}=$ $\mathbb{C}[x] / p(x)$ and $C$-basis $b$ (fixed by $\Phi$ ) and associated $\mathrm{DTT}_{n}$. Let $\phi$ be the representation associated with the model.
Filtering in this model is the multiplication of $h \in \mathcal{A}$ (expressed in the $T$-basis) with $s \in \mathcal{M}$ (expressed in $b$ ) modulo $p$ to yield again a signal expressed in $b$. In coordinates, $h s$ mod $p$ is equivalent to $\phi(h) \mathbf{s}$.

The diagonalization properties of the 16 DTTs are a special case of (11) and can be stated in a unified way. For any filter $h \in \mathcal{A}$,

$$
\operatorname{DTT}_{n} \cdot \phi(h) \cdot \operatorname{DTT}_{n}^{-1}=\operatorname{diag}_{0 \leq k<n}\left(h\left(\alpha_{k}\right)\right)
$$

where the $\alpha_{k}$ are the zeros of $p(x)$. This unifies and explains the result from [18]. Conversely, the $\phi(h)$ are all the matrices diagonalized by DTT. The matrices $\phi(h)$ have in all cases structure: each $\phi(h)$ can be written as the sum of a Toeplitz and a Hankel matrix, up to potential scaling factors. More details are in [6].

As one example, for $\phi(x)$ in (29), we get

$$
\text { DCT- } 2_{n} \cdot \phi(x) \cdot \text { DCT- } 2_{n}^{-1}=\operatorname{diag}_{0 \leq k<n}(\cos (k \pi / n)) .
$$

More generally, the DTTs diagonalize their associated $\phi(x)$ in (30) via (31), which was also observed in [16] (where $\phi(2-$ $2 x)=2 I_{n}-2 \phi(x)$ was considered instead of $\left.\phi(x)\right)$. This also implies that the $\phi(x)$ have pairwise distinct eigenvalues.

Equation (31) also provides the convolution theorems associated with the finite space models.

\section{E. Orthogonal DTTs}

It is well known that the DTTs, as defined in Table III, are "almost orthogonal," which means that after a suitable scaling of rows and columns they become orthogonal. Using ASP, i.e., the knowledge of the DTTs' underlying signal models (24), these scaling factors can be derived as explained in [6] and omitted here due to space limitations.

Another argument (following [16]) for the "almost orthogonality" of the DTTs is that they diagonalize the matrices in (30), which are almost symmetric and have pairwise distinct eigenvalues as mentioned above. For example, DCT-2 diagonalizes the symmetric $\phi(x)$ in (29) and hence can be made orthogonal by a suitable scaling $D$. DCT-2, where $D$ is diagonal.

\section{F. Relationships Between DTTs}

Some DTTs can be translated into each other using sparse matrices. These relationships can be understood and derived once their underlying signal models are known. We explained this in [9] (without using the notion of signal model) and briefly restate the result for completeness. The origin of these relationships is similarity in the signal model, i.e., that two DTTs belong to the same group of four (e.g., $T$-group).

Duality: We observed before that the right boundary conditions for the DTTs are precisely the mirrored versions of the left boundary conditions, a fact that meets our intuition since the DTTs are based on symmetric space models. However, the construction of $\mathcal{A}=\mathcal{M}=\mathbb{C}[x] / p$ for a given DTT (see Theorem 6) deals differently with the left boundary condition (which determines the choice of the base sequence $C$ ) and the right boundary condition (which determines $p$ ); thus, we obtain different DTTs for a given pair of boundary conditions and for its mirrored counterpart. We call such a pair dual. Dual DTTs occur at mirrored positions in Table IV, i.e., at positions $(i, j)$, 
$(j, i), 1 \leq i, j \leq 4$, respectively. The DTTs on the main diagonal are self-dual.

Theorem 8 (Duality Relationship) [9]: Let $\mathrm{DTT}_{n}$ and $\mathrm{DTT}_{n}^{\prime}$ be a pair of dual DTTs. Then

$$
\operatorname{diag}_{0 \leq k<n}\left((-1)^{k}\right) \cdot \mathrm{DTT}_{n}=\mathrm{DTT}_{n}^{\prime} \cdot J_{n}
$$

where $J_{n}$ is an identity matrix with the columns in reversed order. As an important consequence of Theorem 8, dual DTTs have the same arithmetic complexity.

Relationships in Groups of DTTs: Dual DTTs necessarily have the same associated $\mathcal{A}=\mathcal{M}=\mathbb{C}[x] / p$. However, in Table IV, we also have DTTs that are not dual but have the same or similar $\mathcal{M}$, namely those in the same group of four (e.g., $T$-group). An example is given by the DCTs of type 3 and 4 with $\mathcal{M}=\mathbb{C}[x] / T_{n}$.

Further inspection shows that, in each group, all possible left and right boundary conditions are present. The DTTs in one group have (almost) the same module, but with different bases. Thus, we can translate DTTs in the same group into each other using a base change. Further, because of Table II, the resulting base change matrices are sparse, i.e., require only $O(n)$ operations.

Example: DCT, Type 3 and 4: We consider DCT- $3_{n}$ and DCT $-4_{n}$, which are both in the $T$-group, i.e., the associated module is $\mathcal{M}=\mathbb{C}[x] / T_{n}$. The difference is in the choice of basis:

$$
\begin{array}{lll}
\text { DCT} 3_{n}: & \mathbb{C}[x] / T_{n}, & b=\left(T_{0}, \ldots, T_{n-1}\right) \\
\text { DCT} 4_{n}: & \mathbb{C}[x] / T_{n}, & b^{\prime}=\left(V_{0}, \ldots, V_{n-1}\right) .
\end{array}
$$

Using $T_{\ell}=\left(V_{\ell}+V_{\ell-1}\right) / 2$ from Table II and $V_{-1}=V_{0}$, the corresponding base change matrix $S_{n}^{\prime}$ for $b \rightarrow b^{\prime}$ is given by

$$
S_{n}^{\prime}=\frac{1}{2} \cdot\left[\begin{array}{ccccc}
2 & 1 & & & \\
0 & 1 & 1 & & \\
& & \cdot & \cdot & \\
& & & 1 & 1 \\
& & & & 1
\end{array}\right]
$$

We denote the zeros of $T_{n}$ by $\alpha_{k}=\cos (k+1 / 2) \pi / n$. As a consequence of the above, we get the following diagram:

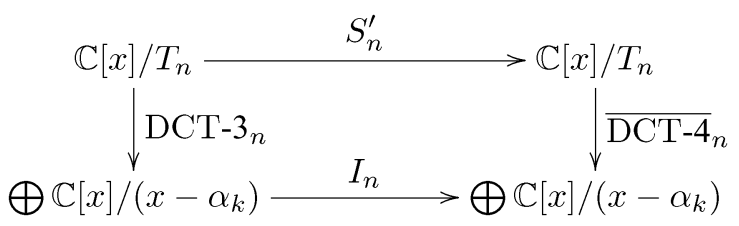

which implies the equation DCT- $3_{n}=\overline{\mathrm{DCT}}-4_{n} \cdot S_{n}^{\prime}$. Note that we have $I_{n}$ in the bottom row of (33) since both DCT-3 = $\overline{\mathrm{DCT}}-3$ and $\overline{\mathrm{DCT}}-4$ are polynomial transforms and thus use the same basis $(1, \ldots, 1)$ in the spectrum. Introducing the scaling diagonal $D_{n}=\operatorname{diag}_{0<k<n}(\cos (2 k+1) \pi /(4 n))$ of the DCT-4 (see Table IV), we get

$$
D_{n} \cdot \mathrm{DCT}-3_{n}=\mathrm{DCT}-4_{n} \cdot S_{n}^{\prime} \text {. }
$$

If desired, this equation can now be further manipulated through transposition or inversion. As an example, one can obtain

$$
S_{n} \cdot \mathrm{DCT}-2_{n} \cdot \frac{1}{2} D_{n}^{-1}=\mathrm{DCT}-4_{n}
$$

where $S_{n}$ is $S_{n}^{\prime}$ with the 2 replaced by 1 in the first entry and without the scaling factor $1 / 2$.

Other Cases: Using this procedure on all DTTs shows that all DTTs of types 1-4 and all DTTs of type 5-8 can be converted into each other using $O(n)$ operations, respectively [9].

\section{Finite SKEW $C$-TRAnsform AND SKEW DTTS}

In this section, we introduce a new class of transforms that is closely related to the DTTs. We call these transforms skew DTTs. More specifically, the skew DTTs correspond to and generalize the DTTs in the $T$-group, i.e., those with associated $\mathcal{A}=\mathcal{M}=\mathbb{C}[x] / T_{n}$, which are the DTTs of type 3 and 4. The first skew DCT (type 3) was introduced in [19].

We introduce the skew DTTs for the following reasons. First, they are interesting from a signal processing point of view. As the DTTs, they are associated with a finite space model, their associated boundary conditions are simple, and their signal extension is sparse even though not monomial.

Second, they are necessary building blocks in the generalradix Cooley-Tukey type DTT algorithms derived in [7].

\section{A. Constructing the Signal Model}

In the finite space models (24), we chose the right boundary condition to ensure a monomial signal extension via Lemma 5. Now we relax this requirement and consider a more general boundary condition for the four signal models in (24) for which $\mathcal{A}=\mathcal{M}=\mathbb{C}[x] / T_{n}$. Namely we generalize to $\mathcal{A}=\mathcal{M}=$ $\mathbb{C}[x] /\left(T_{n}-\cos r \pi\right), r \in \mathbb{Q}, 0 \leq r \leq 1$. For $r=1 / 2$, $\cos r \pi=0$, which is the previous case. Hence, the Fourier transforms will generalize the DTTs in the $T$-group and depend on $r$.

The right boundary conditions associated with $\mathbb{C}[x] /\left(T_{n}-\right.$ $\cos r \pi$ ) depend on the basis $C \in\{T, U, V, W\}$ and can be read off from Table IV:

$$
\begin{aligned}
T_{n} & =\cos r \pi \\
U_{n} & =U_{n-2}+2 \cos r \pi \\
V_{n} & =-V_{n-1}+2 \cos r \pi \\
W_{n} & =W_{n-1}-2 \cos r \pi .
\end{aligned}
$$

In the general case $r \neq 1 / 2$, these boundary conditions lead to no monomial signal extensions, since this property uniquely defines the signal models for the 16 DTTs. However, it is intriguing that the signal extension is "two-monomial," which means that the sum in (7) has at most two summands.

Lemma 9: The module $\mathbb{C}[x] /\left(T_{n}-\cos r \pi\right)$ with $T-, U-, V$-, or $W$-basis has a two-monomial signal extension.

Proof: The proof and the exact form of the signal extension can be found in [6].

Resulting Finite Space Model: We define four skew finite space models parameterized by $r \in Q, 0<r<1$, for $V=\mathbb{C}^{n}$. Namely, for $C \in\{T, U, V, W\}$,

$$
\begin{aligned}
& \mathcal{A}=\mathcal{M}=\mathbb{C}[x] /\left(T_{n}-\cos r \pi\right) \\
& \Phi: \mathbf{s} \mapsto \sum_{0 \leq k<n} s_{k} C_{k} \in \mathcal{M} .
\end{aligned}
$$

As in (24), the natural basis of $\mathcal{A}$ is the T-basis: $\mathcal{A}=\{h=$ $\left.\sum_{0 \leq k<n} h_{k} T_{k}\right\}$, independent of $C$. For $r=1 / 2$, the skew models reduce to their nonskew counterpart in (24). 


\section{B. Spectrum and Fourier Transform: Skew DTTs}

To compute the spectrum and a Fourier transform for the four models (37), we first need to determine the zeros of $T_{n}-\cos r \pi$ and fix a proper ordering.

Lemma 10: Let $r \in Q, 0<r<1$. We have the factorization

$$
T_{n}-\cos r \pi=2^{n-1} \prod_{0 \leq i<n}\left(x-\cos \frac{r+2 i}{n} \pi\right)
$$

which determines the zeros of $T_{n}-\cos r \pi$. We order the zeros as $\alpha=\left(\cos r_{0} \pi, \ldots, \cos r_{n-1} \pi\right)$, such that $0 \leq r_{i} \leq 1$, and $r_{i}<r_{j}$ for $i<j$. The list $\alpha$ is given by the concatenation

$$
\alpha=\bigcup_{0 \leq i<n / 2}\left(\cos \frac{r+2 i}{n} \pi, \cos \frac{2-r+2 i}{n} \pi\right)
$$

for even $n$, and by

$$
\begin{array}{r}
\alpha=\left(\bigcup_{0 \leq i<\frac{n-1}{2}}\left(\cos \frac{r+2 i}{n} \pi, \cos \frac{2-r+2 i}{n} \pi\right)\right) \\
\cup\left(\cos \frac{r+n-1}{n} \pi\right)
\end{array}
$$

for odd $n$. In the particular case of $r=1 / 2$ or $\cos r \pi=0$, we thus have $\alpha=(\cos (i+1 / 2) \pi / n \mid 0 \leq i<n)$ as in Table VII.

Proof: The zeros of $T_{n}-\cos r \pi$ are proved using the closed form of $T_{n}$ in Table VII. The ordering of $\alpha$ is shown by inspection. We omit the details.

In words, the list $\alpha$ arises from the list $\gamma=(\cos (r+$ $2 i) \pi / n \mid 0 \leq i<n)$ in (38) by interleaving the first half of $\gamma$ with the reversed second half of $\gamma$.

Lemma 10 yields the Fourier transform for the models (37). We omit the form (8) and give directly the matrix forms $\mathcal{F}$.

Definition 11 (Skew DTTs): Let $p=T_{n}-\cos r \pi, 0<r<1$, and $\mathcal{A}=\mathcal{M}=\mathbb{C}[x] / p$ with basis $b=\left(C_{0}, \ldots, C_{n-1}\right)$, where $C$ is one of $T, U, V, W$. Let $\alpha=\left(\cos r_{i} \pi\right)_{0 \leq i<n}$ denote the list of zeros of $p$ in the order specified in Lemma 10 . We denote the associated polynomial transforms $\mathcal{P}_{b, \alpha}$ for $\mathcal{M}$ by $\overline{\mathrm{DCT}}_{n}(r)$, $\overline{\mathrm{DST}}-3_{n}(r), \overline{\mathrm{DCT}}-4_{n}(r), \overline{\mathrm{DST}}-4_{n}(r)$, for $C=T, U, V, W$, respectively. Further, we define for each of these four $\overline{\mathrm{DTT}}(r)$ the associated scaled polynomial transforms

$$
\operatorname{DTT}_{n}(r)=\operatorname{diag}_{0 \leq i<n}\left(f\left(\cos r_{i} \pi\right)\right) \cdot \overline{\mathrm{DTT}}_{n}(r)
$$

where $f$ is the scaling function associated with the (ordinary) DTT (see Table IV). We call these transforms skew DTTs. If $r=1 / 2$, then $\overline{\mathrm{DTT}}_{n}(1 / 2)=\overline{\mathrm{DTT}}_{n}$ and $\mathrm{DTT}_{n}(1 / 2)=\mathrm{DTT}_{n}$ in all four cases. In the case of the DCT- $3_{n}(r)={\overline{\mathrm{DCT}}-3_{n}}_{n}(r)$, we will omit the bar for the skew versions. Specifically

$$
\begin{aligned}
\text { DCT- }-3_{n}(r) & =\left[\cos r_{k} \ell \pi\right]_{0 \leq k, \ell<n} \\
\text { DST- }-3_{n}(r) & =\left[\sin r_{k}(\ell+1) \pi\right]_{0 \leq k, \ell<n} \\
\text { DCT- }-4_{n}(r) & =\left[\cos r_{k}(\ell+1 / 2) \pi\right]_{0 \leq k, \ell<n} \\
\operatorname{DST}-4_{n}(r) & =\left[\sin r_{k}(\ell+1 / 2) \pi\right]_{0 \leq k, \ell<n} .
\end{aligned}
$$

As an example, we consider the DCT- $4_{3}(1 / 3)$. Using Lemma 10 , the zeros of $T_{3}-\cos (\pi / 3)=T_{3}-1 / 2$ are given by $\alpha=$ $(\cos (\pi / 9), \cos (5 \pi / 9), \cos (7 \pi / 9))$. We get

$$
\text { DCT }-4_{3}(1 / 3)=\left[\begin{array}{lll}
\cos \frac{1}{18} \pi & \cos \frac{1}{6} \pi & \cos \frac{5}{18} \pi \\
\cos \frac{5}{18} \pi & \cos \frac{5}{6} \pi & \cos \frac{11}{18} \pi \\
\cos \frac{7}{18} \pi & \cos \frac{5}{6} \pi & \cos \frac{1}{18} \pi
\end{array}\right] .
$$

\section{Filtering and Diagonalization Property}

Filtering in the models (37) is multiplication of polynomials $h \in \mathcal{A}, s \in \mathcal{M}$ modulo $p=T_{n}-\cos r \pi$. In coordinates, it becomes the matrix-vector multiplication $\phi(h) \mathbf{s}$, where $\phi$ is the representation associated by the respective model. Convolution theorems are special cases of (11).

As an example, we compute the shift matrix $\phi(x)$ from (30) and (36). Specifically, it is obtained from (30) by adding in the upper right corner $\beta(r)=\cos r \pi$ for DCT-3 $(r)$, and $\beta(r)=$ $2 \cos r \pi$ for the other skew transforms. Hence,

$$
\phi(x)=\frac{1}{2} \cdot\left[\begin{array}{cccccc}
\beta_{1} & 1 & & & & \beta(r) \\
\beta_{2} & 0 & 1 & & & \\
0 & 1 & 0 & . & & \\
& & 1 & \cdot & 1 & \\
& & & \cdot & 0 & \beta_{3} \\
& & & & 1 & \beta_{4}
\end{array}\right] .
$$

The values for the $\beta_{i}$ coincide with the non-skew cases given in Table V. As a consequence, in the four cases

$$
\operatorname{DTT}(r) \cdot \phi(x) \cdot \operatorname{DTT}(r)^{-1}=\operatorname{diag}(\alpha)
$$

where $\alpha$ is the list of zeros of $T_{n}-\cos r \pi$ from Lemma 10.

\section{Translation Into Non-Skew DTTs}

Each of the skew DTTs can be translated into its non-skew counterpart using a sparse $\mathrm{X}$-shaped matrix.

Lemma 12: Let $\mathrm{DTT}_{n}(r)$ be a skew DTT. Then

$$
\operatorname{DTT}_{n}(r)=\operatorname{DTT}_{n} \cdot X_{n}^{(*)}(r)
$$

and

$$
\overline{\mathrm{DTT}}_{n}(r)=\overline{\mathrm{DTT}}_{n} \cdot X_{n}^{(*)}(r)
$$

Here, $X_{n}^{(*)}(r)$ depends on the DTT and takes the following forms, indicated by $* \in\{C 3, S 3, C 4, S 4\}$. In all four cases, if the lines intersect, the numbers are added at the intersecting position.

$$
\begin{aligned}
& X_{n}^{(C 3)}(r)=\left[\begin{array}{ccccc}
1 & 0 & \cdots & \cdots & 0 \\
0 & c_{1} & & & s_{n-1} \\
\vdots & & \ddots & . & \\
\vdots & & . \cdot & \ddots & \\
0 & s_{1} & & & c_{n-1}
\end{array}\right] \\
& X_{n}^{(S 3)}(r)=\left[\begin{array}{ccccc}
c_{1} & & & -s_{n-1} & 0 \\
& \ddots & . & & \\
& . \cdot & \ddots & & \vdots \\
-s_{1} & & & c_{n-1} & 0 \\
0 & \ldots & \ldots & 0 & c_{n}
\end{array}\right]
\end{aligned}
$$


with $c_{\ell}=\cos (1 / 2-r) \ell \pi / n$ and $s_{\ell}=\sin (1 / 2-r) \ell \pi / n$.

$$
X_{n}^{(C 4)}(r)=\left[\begin{array}{cccc}
c_{0}^{\prime} & & & s_{n-1}^{\prime} \\
& \ddots & . & \\
& . \cdot & \ddots & \\
s_{0}^{\prime} & & & c_{n-1}^{\prime}
\end{array}\right]
$$

with $c_{\ell}^{\prime}=\cos (1 / 2-r)(2 \ell+1) \pi /(2 n)$ and $s_{\ell}^{\prime}=\sin (1 / 2-$ $r)(2 \ell+1) \pi /(2 n)$. For DST- $4(r)$, the sines $s_{\ell}^{\prime}$ in $X_{n}^{(C 4)}(r)$ are multiplied by -1 .

Proof: Follows by direct computation, using the definitions of the matrices and $\cos (x) \cos (y)=(\cos (x+y)+\cos (x-$ $y)) / 2$.

Note that the $2 \times 2$ blocks in the translation matrices $X_{n}(r)$ are not rotations. The identities in Lemma 12 enable the inversion of the skew DTTs through the inversion of the ordinary DTTs.

\section{E. Relationships Between Skew DTTs}

All skew DTT $(r)$ share the same associated module, but different bases. Thus, they can be translated into each other by a base change similar to the ordinary DTTs in Section IV-F. As in that section, we consider the skew DCTs, type 3 and 4, as an example. The base change matrix $S_{n}^{\prime}$ we computed in (32) did not depend on the right boundary condition. Thus, the diagram (33) generalizes for arbitrary $r$ to

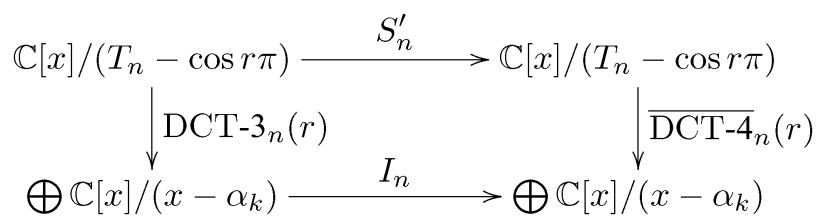

which implies DCT- $3_{n}(r)=\overline{\mathrm{DCT}}_{n}(r) \cdot S_{n}^{\prime}$. The first difference occurs when we extend (40) to the non-polynomial DCT- $4_{n}(r)$, since the scaling diagonal depends on $r$. Let $\alpha=$ $\left(\alpha_{0}, \ldots, \alpha_{n-1}\right)$ denote the zeros of $T_{n}-\cos r \pi$ and $f$ the scaling function of DCT-4 and let $D_{n}(r)=$ $\operatorname{diag}_{0 \leq k<n}\left(f\left(\alpha_{k}\right)\right)$. Then

$$
D_{n}(r) \cdot \mathrm{DCT}-3_{n}(r)=\mathrm{DCT}-4_{n}(r) \cdot S_{n}^{\prime},
$$

which generalizes (34).

In Section IV-F, we continued by inverting this equation to derive the different relationship (35). To do this, we introduce the proper "inverse" skew DTTs, which will also be needed in the DTT algorithms derived in [7]. The definition is motivated by and a generalization of the equations

$$
\begin{aligned}
\text { DCT- }-3_{n}^{-1} & =2 / n \cdot \operatorname{diag}(1 / 2,1, \ldots, 1) \cdot \operatorname{DCT}-2_{n} \\
\text { DST}^{-1} 3_{n}^{-1} & =2 / n \cdot \operatorname{diag}(1,1, \ldots, 1 / 2) \cdot \operatorname{DST}-2_{n} \\
\text { DTTT }_{n}^{-1} & =n / 2 \cdot \operatorname{DTT}_{n}^{T}=n / 2 \cdot \mathrm{DTT}_{n}
\end{aligned}
$$

for DTT $=$ DCT-4, DST-4.

Definition 13 (Inverse Skew DTTs): We define the inverse skew DTTs by

\begin{tabular}{|c|c|c|c|}
\hline $\mathcal{A}=\mathcal{M}$ & $\Phi$ & $\mathcal{F}=\mathcal{P}_{b, \alpha}$ & other $\mathcal{F}$ \\
\hline $\begin{array}{l}\mathbb{C}[x] /\left(x^{2}-1\right) U_{n-2} \\
\mathbb{C}[x] / T_{n} \\
\mathbb{C}[x] /(x-1) W_{n-1} \\
\mathbb{C}[x] /(x+1) V_{n-1} \\
\mathbb{C}[x] /\left(T_{n}-\cos r \pi\right)\end{array}$ & $\mathbf{s} \mapsto \sum s_{k} T_{k}$ & $\begin{array}{l}\text { DCT }-1_{n} \\
\text { DCT- } 3_{n} \\
\text { DCT- }-5_{n} \\
\text { DCT- } 7_{n} \\
\text { DCT- } 3_{n}(r)\end{array}$ & $\begin{array}{l}- \\
- \\
- \\
-\end{array}$ \\
\hline $\begin{array}{l}\mathbb{C}[x] / T_{n} \\
\mathbb{C}[x] / U_{n} \\
\mathbb{C}[x] / V_{n} \\
\mathbb{C}[x] / W_{n} \\
\mathbb{C}[x] /\left(T_{n}-\cos r \pi\right)\end{array}$ & $\mathbf{s} \mapsto \sum s_{k} U_{k}$ & $\begin{array}{l}\overline{\mathrm{DST}}_{3} n \\
\overline{\mathrm{DST}}_{n} \\
\overline{\mathrm{DCT}}_{n} \\
\overline{\mathrm{DST}}_{n} \\
\overline{\mathrm{DST}}_{3}(r)_{n}\end{array}$ & $\begin{array}{l}\text { DST- } 3_{n} \\
\text { DST- } 1_{n} \\
\text { DCT- } 7_{n} \\
\text { DST- } 5_{n} \\
\text { DST- } 3_{n}(r)\end{array}$ \\
\hline $\begin{array}{l}\mathbb{C}[x] /(x-1) W_{n-1} \\
\mathbb{C}[x] / V_{n} \\
\mathbb{C}[x] /(x-1) U_{n-1} \\
\mathbb{C}[x] / T_{n} \\
\mathbb{C}[x] /\left(T_{n}-\cos r \pi\right)\end{array}$ & $\mathbf{s} \mapsto \sum s_{k} V_{k}$ & 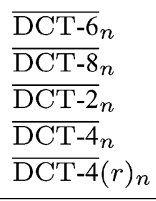 & $\begin{array}{l}\text { DCT- } 6_{n} \\
\text { DCT- } 8_{n} \\
\text { DCT- } 2_{n} \\
\text { DCT- } 4_{n} \\
\text { DCT }-4_{n}(r) \\
\end{array}$ \\
\hline $\begin{array}{l}\mathbb{C}[x] /(x+1) V_{n-1} \\
\mathbb{C}[x] / W_{n} \\
\mathbb{C}[x] / T_{n} \\
\mathbb{C}[x] /(x+1) U_{n-1} \\
\mathbb{C}[x] /\left(T_{n}-\cos r \pi\right)\end{array}$ & $\mathbf{s} \mapsto \sum s_{k} W_{k}$ & $\begin{array}{l}\overline{\mathrm{DST}-}_{n} \\
\overline{\mathrm{DST}}_{n} \\
\overline{\mathrm{DCT}}_{n} \\
\overline{\mathrm{DST}}_{n} \\
\overline{\mathrm{DST}}_{n} \\
(r)_{n}\end{array}$ & $\begin{array}{l}\text { DST- } 8_{n} \\
\text { DST- } 6_{n} \\
\text { DCT- } 4_{n} \\
\text { DST- } 2_{n} \\
\text { DST- } 4_{n}(r)\end{array}$ \\
\hline
\end{tabular}

$$
\begin{aligned}
& \text { iDCT- } 3_{n}(r)=n / 2 \cdot \operatorname{diag}(2,1, \ldots, 1) \cdot \text { DCT- } 3_{n}(r)^{-1} \\
& \text { iDCT }-4_{n}(r)=n / 2 \cdot \text { DCT } 4_{n}(r)^{-1} \\
& \text { iDST- } 3_{n}(r)=n / 2 \cdot \operatorname{diag}(1,1, \ldots, 2) \cdot \text { DST- } 3_{n}(r)^{-1} \\
& \text { iDST }-4_{n}(r)=n / 2 \cdot \operatorname{DST}-4_{n}(r)^{-1} \text {. }
\end{aligned}
$$

TABLE VI

Overview of tHe Finite SPACE Models AND AsSOCIATED FouRIER TRANSFORMS DISCUSSED IN THIS PAPER

Thus, for $r=1 / 2$, we have iDCT- $3_{n}(1 / 2)=$ DCT $-2_{n}$, iDST- $3_{n}(1 / 2)=$ DST $-2_{n}$, iDCT- $4_{n}(1 / 2)=$ DCT $-4_{n}$, iDCT $-4_{n}(1 / 2)=$ DCT $-4_{n}$.

Note that Definition 13 does not provide direct knowledge about the matrix entries of the iDTTs. These, however, can be computed using Lemma 12. For example

$$
\begin{aligned}
& \text { iDCT- } 3_{n}(r)=\left(X_{n}^{(C 3)}(r)\right)^{-1} \cdot \text { DCT- } 2_{n} \\
& \text { iDCT- } 4_{n}(r)=\left(X_{n}^{(C 4)}(r)\right)^{-1} \cdot \text { DCT- } 4_{n}
\end{aligned}
$$

and similarly for DST-3 and DST-4. Note that $\left(X_{n}^{(*)}(r)\right)^{-1}$ has in all four cases the same $\mathrm{x}$-shaped pattern as $X_{n}(r)$. Namely, the four inverses are derived from

$$
\left[\begin{array}{ll}
\cos a & \sin b \\
\sin a & \cos b
\end{array}\right]^{-1}=\frac{1}{\cos (a+b)}\left[\begin{array}{rr}
\cos b & -\sin b \\
-\sin a & \cos a
\end{array}\right]
$$

For example

$$
\begin{array}{r}
\left(X_{n}^{(C 3)}(r)\right)^{-1}=\frac{1}{\cos (1 / 2-r) \pi} \\
\times\left[\begin{array}{ccccc}
c_{n} & 0 & \ldots & \ldots & 0 \\
0 & c_{n-1} & & & -s_{n-1} \\
\vdots & & \ddots & . & \\
\vdots & & . \cdot & \ddots & \\
0 & -s_{1} & & & c_{1}
\end{array}\right] .
\end{array}
$$

Using Definition 13, we can now invert (34) to get a generalization of (35)

$$
S_{n} \cdot \operatorname{iDCT}-3_{n}(r) \cdot \frac{1}{2} D_{n}(r)^{-1}=\operatorname{iDCT}-4(r)_{n}
$$

where $S_{n}$ is the same as in (35). 
TABLE VII

Four Series of Chebyshev Polynomials. In the TRIGONOMETRIC Closed Form $\cos \theta=x$ AND IN THE POWER FORM $\left(u+u^{-1}\right) / 2=x$

\begin{tabular}{llllll}
\hline$C_{n}$ & $n=0,1$ & closed form & power form & symmetry & zeros $(0 \leq k<n)$ \\
\hline$T_{n}$ & $1, x$ & $\cos (n \theta)$ & $\frac{u^{n}+u^{-n}}{2}$ & $T_{-n}=T_{n}$ & $\cos \frac{\left(k+\frac{1}{2}\right) \pi}{n}$ \\
$U_{n}$ & $1,2 x$ & $\frac{\sin (n+1) \theta}{\sin \theta}$ & $\frac{u^{n+1}-u^{-(n+1)}}{u-u^{-1}}$ & $U_{-n}=-U_{n-2}$ & $\cos \frac{(k+1) \pi}{n+1}$ \\
$V_{n}$ & $1,2 x-1$ & $\frac{\cos \left(n+\frac{1}{2}\right) \theta}{\cos \frac{1}{2} \theta}$ & $\frac{u^{n+1 / 2}+u^{-(n+1 / 2)}}{u^{1 / 2}+u^{-1 / 2}}$ & $V_{-n}=V_{n-1}$ & $\cos \frac{\left(k+\frac{1}{2}\right) \pi}{n+\frac{1}{2}}$ \\
$W_{n}$ & $1,2 x+1$ & $\frac{\sin \left(n+\frac{1}{2}\right) \theta}{\sin \frac{1}{2} \theta}$ & $\frac{u^{n+1 / 2}-u^{-(n+1 / 2)}}{u^{1 / 2}-u^{-1 / 2}}$ & $W_{-n}=-W_{n-1}$ & $\cos \frac{(k+1) \pi}{n+\frac{1}{2}}$ \\
\hline
\end{tabular}

\section{OVERVIEW OF Finite Space Models}

In Table VI, we list all the finite space signal models, and their associated Fourier transforms, that we introduced in this paper. The table is divided according to $\Phi$, which is a finite $T-, U-, V-$, or $W$-transform.

In each row, we list in the first two columns the signal model, in the third column the associated unique polynomial Fourier transform, and in the fourth column possibly other relevant Fourier transforms for the model.

Except for the skew DTTs, each of the listed transforms has an orthogonal counterpart, which is obtained by proper scaling of rows or columns.

Table VI, together with Table II in [1] for finite 1-D time models classifies practically all existing 1-D trigonometric transforms, i.e., those transforms that can be expressed using cosines and sines. For each of these transforms, ASP hence provides the associated signal model and with it all basic SP concepts, many of which have not been defined or found before.

\section{CONCLUSION}

This paper shows that a theory of linear signal processing can be developed from a new concept of shift that is different from the standard time shift, namely from the space shift as we call it. Using the algebraic signal processing theory, we derived from this shift appropriate signal models for space signal processing, i.e., filter algebras, signal modules, and " $z$-transforms." In the finite case this approach derived from basic principles the 16 DTTs as Fourier transforms. This interpretation is arguably more satisfying than the original one as asymptotic approximations of the Karhunen-Loève transform (KLT) of a first-order causal Gauss-Markov random process. For a closer investigation of the relationship between KLTs and DTTs and between KLTs and general Fourier transforms in ASP see [6], [20].

By identifying the signal models underlying the DTTs, we also identified their associated notions of " $z$-transform," filtering or convolution, and explained in one framework many of the known properties of the DTTs. In [7], [9] we use the knowledge of these signal models to derive known and novel fast DTT algorithms.

One may wonder which other shifts provide meaningful SP frameworks and ASP is the proper platform to investigate this question. We have done first steps in this direction with a generalization of the space shift (called GNN shift) in [6], and with 2-D space shifts for both the quincunx lattice [21] and the hexagonal lattice [22]. The latter two yield nonseparable 2-D signal models.

\section{APPENDIX I \\ ChebysheV Polynomials}

Chebyshev polynomials, and the more general class of orthogonal polynomials, have many interesting properties and play an important role in different areas of mathematics, including statistics, approximation theory, and graph theory. An excellent introduction to the theory of orthogonal polynomials can be found in the books of Chihara, Szegö, and Rivlin [23]-[25]. In this section we give the main properties of Chebyshev polynomials that we will use in this paper.

We call every sequence $C=\left(C_{n}\right)_{n \in \mathbb{Z}}$ of polynomials that satisfies the three-term recurrence

$$
C_{n+1}(x)=2 x C_{n}(x)-C_{n-1}(x)
$$

a sequence of Chebyshev polynomials ( $C$ stands for Chebyshev). Using (43), the sequence $C$ is uniquely determined by the initial polynomials $C_{0}, C_{1}$. The most important-and commonly known - are the Chebyshev polynomials of the first kind, denoted by $C_{n}=T_{n}$ and determined by $T_{0}=1$ and $T_{1}=x$. We provide a few examples:

$$
\begin{array}{cccccc}
T_{-2} & T_{-1} & T_{0} & T_{1} & T_{2} & T_{3} \\
\hline 2 x^{2}-1 & x & 1 & x & 2 x^{2}-1 & 4 x^{3}-3 x
\end{array}
$$

For $x \in[-1,1], T_{n}$ can be written in closed form as

$$
T_{n}=\cos n \theta, \quad \cos \theta=x .
$$

The closed form exhibits the symmetry property $T_{-n}=T_{n}$, $n \in \mathbb{Z}$, and can be used to derive the zeros of $T_{n}$. We will occasionally use another parameterization of $T_{n}$, which we call power form, given by

$$
T_{n}=\frac{u^{n}+u^{-n}}{2}, \quad \frac{u+u^{-1}}{2}=x .
$$

By substituting $u=e^{j \theta}$ we obtain (44).

In this paper, we also consider the Chebyshev polynomials of the second, third, and fourth kind, denoted by $U_{n}, V_{n}, W_{n}$, respectively, that arise from $C_{0}=1$ and different choices of $C_{1}$. Each of these sequences exhibits a symmetry property and possesses parameterized forms. These properties are summarized in Table VII.

In addition, we will need the following properties that are shared by all sequences of Chebyshev polynomials including $T, U, V, W$ (see [23]).

Lemma 14: Let $C=\left(C_{n}\right)_{n \in \mathbb{Z}}$ be a sequence of Chebyshev polynomials. Then the following holds: 
i) the sequence $C$ is determined by any two successive polynomials $C_{n}, C_{n+1}$;

ii) $\operatorname{deg}\left(C_{0}\right)=0, \operatorname{deg}\left(C_{1}\right)=1 \Rightarrow \operatorname{deg}\left(C_{n}\right)=n$, for $n \geq 0$;

iii) $C_{n}=C_{1} \cdot U_{n-1}-C_{0} \cdot U_{n-2}$;

iv) $T_{k} \cdot C_{n}=\left(C_{n+k}+C_{n-k}\right) / 2$.

\section{REFERENCES}

[1] M. Püschel and J. M. F. Moura, "Algebraic signal processing theory: Foundation and 1-D time," IEEE Trans. Signal Process., vol. 56, no. 8, Aug. 2008.

[2] N. Ahmed, T. Natarajan, and K. R. Rao, "Discrete cosine transform," IEEE Trans. Comput., vol. C-23, no. 1, pp. 90-93, Jan. 1974.

[3] K. R. Rao and P. Yip, Discrete Cosine Transform: Algorithms, Advantages, Applications. New York: Academic Press, 1990.

[4] Z. Wang and B. R. Hunt, "The discrete W transform," Appl. Math. Comput., vol. 16, pp. 19-48, 1985.

[5] R. M. Gray, Probability, Random Processes, and Ergodic Properties. New York: Springer-Verlag, 1988.

[6] M. Püschel and J. M. F. Moura, "Algebraic Signal Processing Theory," [Online]. Available: http://arxiv.org/abs/cs.IT/0612077

[7] M. Püschel and J. M. F. Moura, "Algebraic signal processing theory: Cooley-Tukey type algorithms for DCTs and DSTs," IEEE Trans. Signal Process., vol. 56, no. 4, pp. 1502-1521, Apr. 2008.

[8] Y. Voronenko and M. Püschel, "Algebraic signal processing theory: Cooley-Tukey type algorithms for real DFTs," IEEE Trans. Signal Process., to be published.

[9] M. Püschel and J. M. F. Moura, "The algebraic approach to the discrete cosine and sine transforms and their fast algorithms," SIAM J. Comput., vol. 32, no. 5, pp. 1280-1316, 2003.

[10] G. Steidl and M. Tasche, "A polynomial approach to fast algorithms for discrete Fourier-cosine and Fourier-sine transforms," Math. Comput., vol. 56, no. 193, pp. 281-296, 1991.

[11] T. Kailath and V. Olshevsky, "Displacement structure approach to discrete trigonometric transform based preconditioners of G. Strang and T. Chan type," Calcolo, vol. 33, pp. 191-208, 1996.

[12] E. Feig and M. Ben-Or, "On algebras related to the discrete cosine transform," Linear Algebra Appl., vol. 266, pp. 81-106, 1997.

[13] N. Jacobson, Basic Algebra I. San Francisco, CA: Freeman, 1974.

[14] P. A. Fuhrman, A Polynomial Approach to Linear Algebra. New York: Springer-Verlag, 1996.

[15] J. M. F. Moura and N. Balram, "Recursive structure of noncausal Gauss Markov random fields," IEEE Trans. Inf. Theory, vol. 38, no. 2, pp. 334-354, 1992.

[16] G. Strang, "The discrete cosine transform," SIAM Rev., vol. 41, no. 1, pp. 135-147, 1999.

[17] S. A. Martucci, "Symmetric convolution and the discrete sine and cosine transforms," IEEE Trans. Signal Process., vol. 42, no. 5, pp. 1038-1051, May 1994.

[18] V. Sánchez, P. García, A. M. Peinado, J. C. Segura, and A. J. Rubio, "Diagonalizing properties of the discrete cosine transforms," IEEE Trans. Signal Process., vol. 43, no. 11, pp. 2631-2641, Nov. 1995.

[19] M. Püschel, "Cooley-Tukey FFT like algorithms for the DCT," in Proc. Int. Conf. Acoustics, Speech, Signal Processing (ICASSP), 2003, vol. 2, pp. 501-504.

[20] J. M. F. Moura and M. G. S. Bruno, "DCT/DST and Gauss-Markov fields: Conditions for equivalence," IEEE Trans. Signal Process., vol. 46, no. 9, pp. 2571-2574, Sep. 1998.

[21] M. Püschel and M. Rötteler, "Fourier transform for the spatial quincunx lattice," in Proc. Int. Conf. Image Processing (ICIP), 2005, vol. 2, pp. 494-497.

[22] M. Püschel and M. Rötteler, "Algebraic signal processing theory: 2-D hexagonal spatial lattice," IEEE Trans. Image Process., vol. 16, no. 6, pp. 1506-1521, 2007.

[23] T. S. Chihara, An Introduction to Orthogonal Polynomials. New York: Gordon and Breach, 1978.

[24] G. Szegö, Orthogonal Polynomials, 3rd ed. Providence, RI: Amer. Math. Soc. Colloq. Publ., 1967.

[25] T. J. Rivlin, The Chebyshev Polynomials. New York: Wiley Interscience, 1974.

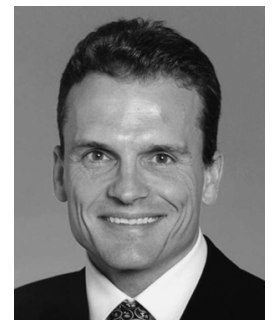

Markus Püschel (M'99-SM'05) received the Diploma (M.Sc.) degree in mathematics and the Ph.D. degree in computer science, both from the University of Karlsruhe, Germany, in 1995 and 1998, respectively.

From 1998 to 1999, he was a Postdoctoral Researcher in the Mathematics and Computer Science Department, Drexel University, Philadelphia. Since 2000, he has been with Carnegie Mellon University (CMU), Pittsburgh, PA, where he is an Associate Research Professor of electrical and computer engineering. His research interests include signal processing theory/software/hardware, scientific computing, compilers, applied mathematics, and algebra.

$\mathrm{He}$ is an Associate Editor for the IEEE TRANSACTIONS on Signal PROCESSING and was an Associate Editor for the IEEE Signal PROCESSING LETTERS. He was also a Guest Editor of the Journal of Symbolic Computation and the Proceedings of the IEEE. He holds the title of Privatdozent of Applied Informatics at the Department of Computer Science, University of Technology, Vienna, Austria, and was awarded (with J. Moura) the CMU College of Engineering Outstanding Research Award.

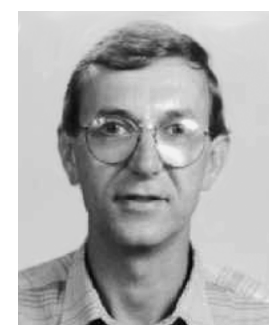

José M. F. Moura (S'71-M'75-SM'90-F'94) received the Engenheiro Electrotécnico degree from Instituto Superior Técnico (IST), Lisbon, Portugal, in 1969 and the M.Sc., E.E., and D.Sc. degrees in electrical engineering and computer science from the Massachusetts Institute of Technology (M.I.T.), Cambridge, in 1973 and 1975, respectively.

$\mathrm{He}$ is a Professor of electrical and computer engineering and, by courtesy, of biomedical engineering at Carnegie Mellon University (CMU), Pittsburgh, PA, where he is a Founding Codirector of the Center for Sensed Critical Infrastructures Research (CenSCIR). He was on the faculty at IST from 1975 to 1984 and has held visiting faculty appointments at the Massachusetts Institute of Technology (MIT), Cambridge, in 1984-1986, 1999-2000, and 2006-2007 and was a research scholar at the University of Southern California (USC) during the summers of 1978 to 1981. In 2006, he founded the Information and Communications Technologies Institute, a joint venture between CMU and Portugal, which he codirects and which manages the CMU-Portugal education and research program www.icti.cmu.edu. His research interests include statistical and algebraic signal processing, image, bioimaging, and video processing, and digital communications. He has published over 300 technical journal and conference papers, is the coeditor of two books, holds six patents on image and video processing, and digital communications with the U.S. Patent Office, and has given numerous invited seminars in the United States and European universities and industrial and government laboratories.

Dr. Moura is the President of the IEEE Signal Processing Society (SPS), previously serving as Vice-President for Publications (2000-2002), Editor-in-Chief for the IEEE TRANSACTIONS IN SIGNAL PROCESSING (1975-1999), interim Editor-in-Chief for the IEEE SigNAL PROCESSING LETTERS (December 2001-May 2002), founding member of the Bioimaging and Signal Processing (BISP) Technical Committee, and member of several other technical committees. He was Vice-President for Publications for the IEEE Sensors Council from 2000 to 2002 and is or was on the Editorial Board of several journals, including the IEEE PROCEEDINGS, the IEEE Signal Processing Magazine, and the ACM Transactions on Sensor Networks. From 2002 to 2003, he chaired the IEEE TAB Transactions Committee that joins the more than 80 Editors-in-Chief of the IEEE Transactions and served on the IEEE TAB Periodicals Review Committee from 2002 to 2006. He is on the Steering Committee of the International Conference on Information Processing and Sensor Networks (IPSN) and was on the Steering Committee of the International Symposium on BioImaging (ISBI) and has been on the program committee of over 35 Conferences and Workshops. He was on the IEEE Press Board from 1991 to 1995. He is a Fellow of the American Association for the Advancement of Science (AAAS) and a corresponding member of the Academy of Sciences of Portugal (Section of Sciences). He was awarded the 2003 IEEE Signal Processing Society Meritorious Service Award, in 2000 the IEEE Millennium Medal, in 2006 an IBM Faculty Award, and in 2007 the CMU College of Engineering Outstanding Research Award (with M. Püschel). He is affiliated with several IEEE societies, Sigma Xi, AMS, AAAS, IMS, and SIAM. 